\title{
Influence de la nature lithologique et des structures géologiques sur la qualité et la dynamique des eaux souterraines dans les hauts plateaux de l'Ouest-Cameroun
}

\author{
A. NONO ${ }^{1 *}$, J. D. H. LIKENG $^{1}$, H. WABO ${ }^{2}$, G. TABUE YOUMBI ${ }^{2}$ et S. BIAYA ${ }^{2}$ \\ ${ }^{1}$ Département des Sciences de la Terre, Faculté des Sciences B.P.67, Université de Dschang, \\ Dschang, Cameroun. \\ ${ }^{2}$ Département des Sciences de la Terre, Faculté des Sciences B.P.812, Université de Yaoundé I, \\ Yaoundé, Cameroun. \\ *Corresponding author, E-mail: alex_nono2000@yahoo.fr
}

\section{RESUME}

Les Hauts plateaux de l'Ouest-Cameroun constituent une vaste zone de hautes terres où de nombreuses populations consomment de l'eau à l'état naturel. Le but de cette étude est d'établir si possible un lien entre les faciès, les structures géologiques, la productivité et la qualité chimique des eaux de consommation. Les méthodes géologiques (cartographie de terrain), la photo-interprétation, les sondages géoélectriques et les analyses chimiques (par volumétrie, colorimétrie et émission de flammes) ont été utilisés. Le site d'étude est un vaste ensemble constitué d'un socle granito-gneissique (gneiss, granitoïdes, amphibolites), recouvert par un manteau volcanique (basaltes, trachytes, rhyolites, ignimbrites), mis en place selon la tectonique de la Chaîne Panafricaine Nord-Equatoriale et de la Ligne Volcanique du Cameroun. Les gneiss et migmatites sont foliés (plan de foliation N25-75E, axe des plis N30-40E et N60-70E), les orthogneiss et amphibolites ont une schistosité variable (N-S à N60E) avec pendage variable (30 à 90 vers le SSW, SW et ESE). La couverture volcanique très répandue ( $3 / 5$ de la superficie) est par endroit fortement altérée et latéritisée. La concordance entre le tracé des cours d'eau, l'allongement des reliefs (NE-SW, NW-SE, et E-W), l'orientation des structures des roches (schistosité, foliation), les diaclases (N20-40E, N130-140E) et les linéaments (N20-40E, N70-80E et N150-160E) favorise l'infiltration des eaux et offre des potentialités hydrogéologiques. Les eaux sont chlorurées calciques et magnésiennes, bicarbonatées calciques et magnésiennes et enfin bicarbonatées sodiques et potassiques, sans liaison aux faciès pétrographiques ni à leur âge. Les minéralisations liées au faciès lithologiques, à la profondeur des nappes et au degré d'altération des roches sont en général faibles $(\leq 587$ $\mathrm{mg} / \mathrm{l})$, les plus fortes valeurs étant obtenues dans les formations basaltiques. La productivité des forages (jusqu'à $20 \mathrm{~m}^{3} / \mathrm{h}$ ) est liée au degré de fracturation et à la nature de la roche.

(C) 2009 International Formulae Group. All rights reserved.

Mots clés: Hauts plateaux, Ouest-Cameroun, faciès, minéralisations, productivité.

\section{INTRODUCTION}

Les Hauts plateaux de l'OuestCameroun forment un vaste ensemble constitué des hautes terres (plateau Bamoun, monts Bambouto, Bamenda, Oku et Bana) et de plaines (Noun et Ndop). Ils sont constitués de formations superficielles (alluvions, éluvions et altérites), des formations volcaniques (basaltes, trachytes, phonolites, rhyolites, tufs, ignimbrites) et du socle granito-gneissique.

La nature des formations géologiques oriente la qualité des eaux souterraines (Djeuda, 1987; Furry, 1997; Derron, 1999), qui au cours de leur trajet souterrain, à travers les fissures et en fonction du temps qu'elles passent au contact de ces formations géologiques, échangent leurs ions avec ceux 
contenus dans les roches. L'altération des minéraux des roches libèrent aussi dans les eaux des éléments mobiles (alcalins et alcalino-terreux) et les éléments traces (Salbu et Steinnes, 1995). Ces éléments, notamment $\mathrm{Pb}^{2+}$ et $\mathrm{Cu}^{2+}$ forment des complexes organométalliques en présence de matière organique (Jackson, 1998). L'oxydation et la précipitation de certains éléments se déroulent lors de l'abaissement du niveau piézométrique. C'est le cas du fer (Temgoua et al., 2005), de la silice et du calcium (Nahon, 1976). Trois principes peuvent être proposés pour l'interaction eau-roche silicatée (Drever et Zobrist, 1992):

1) la composition chimique des eaux est contrôlée par l'altération des minéraux primaires de la roche en phases secondaires;

2) la formation des phases secondaires est déterminée par la composition des solutions;

3) l'altération chimique est sélective: les minéraux ne contribuent pas portionnellement à leur abondance dans la roche à la composition des eaux.

Le problème d'approvisionnement en eau potable se pose avec acuité dans les villes camerounaises où les besoins ne sont que très partiellement satisfaits à cause de la faible extension du réseau de distribution, la forte pression démographique et les faibles revenues d'une importante frange de la population. La situation est plus alarmante dans les zones périurbaines et rurales (cas des Hauts plateaux de l'Ouest-Cameroun) où les populations recourent aux eaux de pluies, des puits, des sources et des forages dont la qualité et la quantité ne sont pas toujours assurées.

L'Etat à travers la Société Nationale des eaux (SNEC), devenue Camerounaise des eaux (CDE) et CAMWATER, couvre moins de $40 \%$ de la demande en eau potable dans tous les centres urbains où elle est présente. En zone rurale, les programmes d'hydraulique villageoise ont permis la réalisation de plusieurs forages afin de faciliter l'accès en eau potable des populations. Malgré la réalisation de ces points d'eau dans les villages, le nombre moyen d'habitants à approvisionner en eau potable est encore élevé (DNS, 1999). Une bonne tranche de la population s'approvisionne encore dans les sources, les puits, et les cours d'eau dont la qualité d'eau n'est pas toujours connue; cette qualité est d'ailleurs rarement déterminée, même dans le cas de certains ouvrages d'hydraulique villageoise (Djeuda et al., 2001). Dans la province de l'Ouest Cameroun, le ratio est de 2775 habitants par ouvrage d'eau (MINMEE/DRH, 1997).

Il se pose donc un problème du choix de la source à aménager et de la méthode fiable d'implantation de forages productifs et de bonne qualité, d'où l'importance de thème d'étude qui est une innovation. Ainsi, les travaux sur l'influence de la nature lithologique et des structures géologiques sur la qualité des eaux de source (Nono et al., 2001, 2004; Biaya, 2002; Nono et al., 2008) montrent que dans les nappes d'altération, les eaux sont dans un état plus ou moins proche de l'équilibre. Pour ces auteurs, le problème majeur rencontré dans les eaux de source et des forages est la présence des fortes teneurs en fer.

La présente étude a pour but de trouver si possible après un examen géologique, géophysique et hydrogéochimique, un lien entre faciès géologiques, productivité des sources et forages, faciès chimiques et minéralisations des sources et forages en liaison avec les normes de potabilité, le fer étant préoccupant dans la mesure où son dépôt sous forme d'oxydes peut nuire à l'environnement (dépôts boueux), obstruer les canalisations, rendre l'eau impropre à la consommation, surtout en zone rurale où il n'y a pas de traitement chimique préalable.

\section{MATERIELS ET METHODES \\ Le site expérimental \\ Cadre géographique}

Les hauts plateaux de l'OuestCameroun (Figure 1) sont situés entre $09^{\circ} 55^{\prime}$ et $11^{\circ} 30^{\prime}$ de longitude Est et $5^{\circ}$ et $6^{\circ} 10^{\prime}$ de latitude Nord. Ce grand ensemble a des altitudes variant entre $1000 \mathrm{~m}$ dans la plaine du Noun et $2740 \mathrm{~m}$ au Mont Meletan dans les Bambouto avec des escarpements d'environ $200 \mathrm{~m}$ de dénivellation (Foréké, Bandjoun, Batié). La géomorphologie d'ensemble est constituée de collines surbaissées, aplanies par l'érosion et les vallées à fond plat dans lesquelles circulent les cours d'eau permanents et intermittents (Regnoult, 1986). La végétation rencontrée est une savane humide avec des plantes hydrophiles et hydrophobes. Les sols sont de types 
ferrallitiques, hydromorphes et peu évolués (Segalen, 1967). L'hydrographie est marquée par des portions rectilignes signe d'un contrôle structural et par de nombreuses chutes et cascades. Les deux plus grands collecteurs de la région sont le Noun et le Nkam. Le climat est de type tropical humide tempéré par l'altitude, avec une pluviométrie moyenne annuelle de $1700 \mathrm{~mm}$ d'eau. La température moyenne annuelle oscille autour de $21^{\circ} \mathrm{C}$.

\section{Cadre géologique et hydrogéologique régional}

Les hauts plateaux de l'ouest Cameroun sont constitués par un socle granito-gneissique panafricain que recouvre un manteau volcanique.

Le socle des hauts plateaux de l'OuestCameroun est essentiellement constitué de granulites hétérogènes (gneiss de haut degré), d'amphibolites et de granitoïdes très répandus dont certains forment des larges plutons tels que la syénite de Bangangté, les charnokites

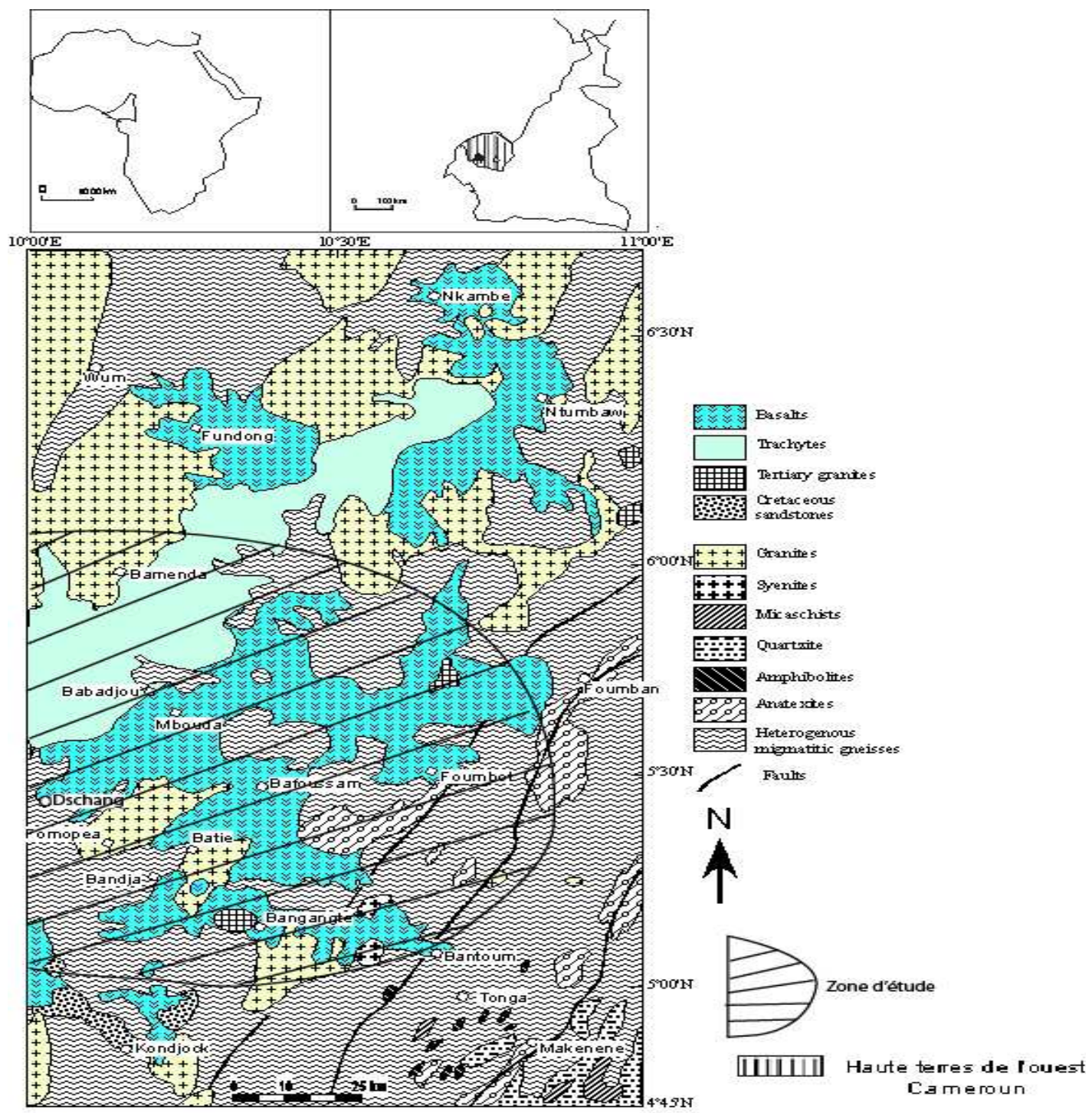

Figure 1: Localisation des hauts plateaux de l'Ouest-Cameroun et de la zone d'étude. Le fond géologique est une compilation de Weecksteen (1957), Dumort (1968) et Peronne (1969), modifié et complété par Nzolang (2005). 
de Bandja et le granite de Batié. Ces granitoïdes correspondent aux «granites circonscrits »d'après Bessoles et Trompette (1980) et sont recouvertes par un complexe volcanique cénozoïque essentiellement basaltique et trachytique (Monts Bambouto, Bamenda et Bangou) et des complexes annulaires anorogéniques (Mont Bana = plutovolcan) qui font partie de la Ligne Volcanique du Cameroun (Déruelle et al., 1991).

Les granitoïdes se sont mis en place suivant la tectonique régionale SW-NE, comme l'attestent le parallélisme entre la schistosité ou fabriques magmatiques des granitoïdes et la schistosité ou foliation dans l'encaissant métamorphique, ainsi que l'allongement des massifs parallèlement à la tectonique régionale (Nzolang, 2005). Dans le secteur de Bantoum (au Sud de Banganté) l'évolution structurale comprend deux phases de déformation; $D_{1}$ tangentielle et $D_{2}$ cisaillante. La $\mathrm{D}_{1}$ est caractérisée dans les granitoïdes par une intense schistosité mylonitique $\mathrm{N} 20-35^{\circ} \mathrm{E}$ souvent associée à une structure S/C plus discrète (Berthé et al., 1979). Dans les gneiss et les amphibolites, $D_{1}$ correspond à une foliation et une schistosité semblables à celles des granitoïdes, des plis isoclinaux à plans axiaux orientés $\mathrm{N} 60-70^{\circ} \mathrm{E}$ et des élongations minérales plongeant à moins de $30^{\circ}$ vers le Sud. L'organisation des plans de foliation et de schistosité les intègre bien dans les grands plis régionaux (antiformes et synformes) plongeant de 40-60 ${ }^{\circ}$ vers le $\mathrm{NE}$ et $\mathrm{SW}$. La phase $\mathrm{D}_{2}$ ne s'observe que dans les roches métamorphiques et se caractérisent par des plans cisaillant N10$20^{\circ} \mathrm{E}$, larges de $5-40 \mathrm{~cm}$, majoritairement sénestres, souvent remplis de leucosome et des plissements variés affectant les gneiss et la schistosité de plan axial associée.

Les roches du socle de la région de Foumban sont constituées d'une unité granitogneissique associée à des roches basiques (amphibolite et monzodiorite) (Njonfang et al., 1998). Le complexe néoprotérozoïque de Ngondo (Tagné Kamga et al., 1999; Tagné Kamga, 2003) est un pluton constitué de trois roches intrudées successivement dans un gneiss à hornblende et biotite: roches basiques à intermédiaires (diorite, granodiorite et grabbro), granites microgrenus et granites porphyroïdes. Ces roches hyperpotassiques, calco-alcalines à subalcalines se sont syntectoniquement mise en place dans la zone de grand cisaillement ductile $\mathrm{N} 30^{\circ} \mathrm{E}$ pendant la phase $\mathrm{D}_{2}$.

Le complexe plutonique de Bandja (Nguiessi et Vialette, 1994) est constitué d'une unité mylonitique comprenant des roches charnokitiques, des orthogneiss et des gneiss lités et une unité moins déformée constituée de granites et monzonites. Leur mise en place syntectonique permet de dater les principales phases de déformation du panafricain : synD ${ }_{1}$ à $640 \mathrm{Ma}(\mathrm{U}-\mathrm{Pb}-\mathrm{Zr})$ dans les charnokites et $557 \mathrm{Ma}(\mathrm{Rb}-\mathrm{Sr})$ dans les granites ainsi leur mise en place tarditectonique $\left(\mathrm{synD}_{3}\right)(\mathrm{Nguessi}$ et al., 1997).

Le massif de Batié (Talla, 1995) est constitué de monzodiorite, granodiorite et de granite à hornblende et biotite alors que le massif de Fomopéa (Kwékam, 1993) est essentiellement formé de granites à hornblende et biotite, associés aux granites à deux micas et des roches basiques (diorites, métadiorires et amphibolites). Ces deux massifs ont des caractéristiques géochimiques similaires (méta-alumineux, calco-alcalin et hyperpotassique) et se sont mis en place à 576 +/- $24 \mathrm{Ma}$ et 575 +/- 17Ma respectivement (datation $\mathrm{Rb}-\mathrm{Sr}$ ).

Le pluton syénitique de Bangangté (Tchouankoué, 1992) est constitué de syénite à gros grains et de monzonite à pyroxène et amphibole montrant des tendances hyperpotassique, alcaline et méta-alumineux. Ils sont datés de 630-606 Ma (datation Rb-Sr).

Les amphibolites et les gneiss (surtout riches en plagioclases) datés du paléoprotérozoïque (1,7-2,3 Ga), ont des caractéristiques géochimiques indicatrices des précurseurs de compositions basaltiques avec des affinités des MORB et des basaltes intraplaques (Nzolang, 2005).

Le massif pluto-volcanique de Bana (Nana, 1988) est intrusif dans le complexe métamorphique panafricain de base et les basaltes de plateau datés probablement de l'éocène-oligocène. De forme annulaire et d'environ $6 \mathrm{~km}$ de diamètre, le massif comprend dans la suite volcanique des mugéarites, benmoréites, rhyolites et ignimbrites rhyolitiques alors que les roches plutoniques sont constituées de leucograbbros, monzodiorites, monzonites et des granites à tendance hyperalunimeuse et hyperalcaline. Les travaux pétrologiques et géochimiques 
(Kuepouo, 2004; Kuepouo et al., 2006) ont permis de distinguer deux séries magmatiques: une série alcaline et une série subalcaline (transitionnnelle). La série alcaline se limite aux basanites, basalte à olivine et hawaiites alors que la série subalcaline forme la principale unité géologique et comprend des roches extrusives et intrusives: basaltes à plagioclase, benmoréite, trachyte comenditique, comendite et pantellérite pour les roches extrusives, syénodiorite et syénodiorite à quartz, granites à arfversonite/aegyrine, granite à biotite et hornblende (métalumineux) et des granites à biotite (légèrement hyperalumineux) pour les roches intrusives. La datation $\mathrm{Rb}-\mathrm{Sr}$ sur roche totale donne un âge de $30 \mathrm{Ma}$ (Lassere, 1978) alors que la méthode $\mathrm{K}-\mathrm{Ar}$ donne $30 \mathrm{Ma}$ pour la phase transitionnelle (Kuepouo et al., 2006).

Le massif volcanique des Bambouto constitue le troisième volcan de la Ligne Volcanique du Cameroun en importance après les Monts Cameroun et Manengouba. Il s'agit d'un volcan polygénique (Saluvio et al., 2000) dont le sommet est occupé par deux caldéras d'effondrement emboîtées (Weeksteen, 1954; Tchoua, 1973). Les produits émis sont outre les laves basiques (basaltes aphyriques latéritisés et basaltes porphyriques), les laves plus ou moins différenciées (trachytes et phonolites) (Youmen, 1994; Youmen et al., 2005; Nni et Nyobé, 1995), des ignimbrites trachytiques et rhyolitiques (Tchoua, 1968 et Dumort, 1968) et des scories anté-ignimbrites (Nono et al., 2004a).

Le massif volcanique de Bangou (44,7 Ma datation ${ }^{40} \mathrm{~K}-{ }^{40} \mathrm{Ar}$ ) est le volcan le plus ancien daté de la Ligne du Cameroun (Fosso et al., 2005). Il s'est édifié en deux phases à la suite d'un volcanisme à dominance fissurale: l'épisode $\mathrm{E}_{1}(44,7 \mathrm{Ma})$ est caractérisée par l'épanchement de laves peu évoluées (basaltes et mugéarites) qui affleurent aujourd'hui en buttes et replats au centre du massif, alors que l'épisode $E_{2}(44,5-43,1 \pm 1 \mathrm{Ma})$ est marquée par une série de laves plus différenciées (trachytes et rhyolites) à la périphérie du massif (Fosso, 1999). Il est à noter en plus des laves de ces deux épisodes, des épanchements de basaltes alcalins de Bandjoun (38 Ma) au Nord et de basaltes alcalins pliocènes plus récents $(4,39 \pm 0,11 \mathrm{Ma})$ recouvrant les laves de l'épisode $\mathrm{E}_{2}$. Dans l'ensemble, toutes les laves des Monts Bangou et ses environs sont très altérées, latéritisées à toutes les altitudes, largement entaillées par l'érosion et recouvertes par une importante végétation. Sur le plan hydrogéologique, très peu de travaux ont été faits sur les hauts plateaux de l'Ouest-Cameroun (Tella, 1999; Nono et al., 2001, 2004b, 2004c). Le massif des Bambouto constitue le second château d'eau du Cameroun après l'Adamaoua. De plus la forte pluviométrie (1700-2500 mm), l'intense activité tectonique (Fracturation), la présence d'innombrables et épais affleurements de tufs et de basaltes prismés et l'altération parfois très poussée des roches font des hauts plateaux de l'Ouest-Cameroun une zone très favorable à la formation des nappes d'eau souterraine qui assurent la régularisation naturelle des débits de saison sèche des cours d'eau des bassins versants du Wouri, de la Sanaga (Dubreuil et al., 1975), de la Bénoué inférieure (Katsena Ala et Donga) et de la Manyu.

\section{Méthodes géologiques}

Elles permettent d'inventorier, de caractériser les types lithologiques, les types de déformations et de mettre en exergue les contacts anormaux présents dans la région, surtout dans les environs des sources et forages à étudier. Elles s'effectuent par des observations et mesures directes sur le terrain suivi d'échantillonnages pour la réalisation des analyses chimiques et des lames minces nécessaires pour les observations microscopiques. Le matériel utilisé est la boussole, le mètre ruban, le marteau et la carte topographique.

\section{Photo interprétation}

Elle utilise les photos aériennes et un stéréoscope pour ressortir les linéaments (lignes résultant des alignements morphostructuraux, des alignements naturels d'arbres, de la disposition des branches des cours d'eau du réseau hydrographique ou de la rectitude des contours géomorphologiques), base de l'établissement de la carte linéamentaire et de fracturation, nécessaire aux traînées et sondages géophysiques.

\section{Méthodes géoélectriques}

Après la mise en évidence grâce à la cartographie et la photogéologie des grandes 
structures (fractures) et en fonction de la morphologie du site, la géophysique peut se mettre en place directement sous forme de profilage (traînées) et de sondage électriques qui permettent respectivement de relever les anomalies latérales et verticales du sol. Le dispositif utilisé est conforme à la norme Schlumberger.

La méthode géoélectrique consiste brièvement à injecter dans le sol un courant continu par l'intermédiaire de deux électrodes A et B (extérieures dites électrodes de courant) et à mesurer la différence de potentiel aux bornes de deux électrodes $\mathrm{M}$ et $\mathrm{N}$ (intérieures dites électrodes de potentiel). Connaissant le facteur de forme $\mathrm{K}$ (distance entre les différentes électrodes), on arrive à déterminer la résistivité apparente des formations géologiques sous-jacentes et à apprécier leurs variations latérales. Les forages existants servent de calage.

\section{Méthodes d'analyse physico-chimique des eaux souterraines}

La technique d'échantillonnage sur le terrain a consisté au rinçage trois fois des bouteilles PVC de 0,501 préalablement lavées et nettoyées à l'eau distillée avec l'eau à prélever et au prélèvement de deux échantillons d'eau par point; un des échantillons est filtré et le filtrat a reçu $1,5 \mathrm{ml}$ d'acide nitrique à $0,1 \mathrm{~N}$ (pour stabiliser les métaux lourds) et l'autre est destiné à l'analyse des anions. Les échantillons remplis à ras bords ont été refermés et soigneusement rangés dans un carton, puis acheminés au laboratoire dans les 48 heures qui ont suivi.

Les différentes méthodes utilisées pour la détermination des teneurs des éléments chimiques sont: la méthode volumétrique pour le dosage des éléments $\mathrm{Ca}^{2+}, \mathrm{Mg}^{2+}, \mathrm{Cl}^{-}$, $\mathrm{HCO}_{3}^{-}$; la méthode colorimétrique pour le dosage du $\mathrm{Fe}^{3+}, \mathrm{SiO}_{2}, \mathrm{Al}^{3+}$ et le dosage par émission de flamme des éléments $\mathrm{Na}^{+}$et $\mathrm{K}^{+}$. Les analyses ont été effectuées au Laboratoire de Chimie Minérale de l'Université de Yaoundé 1 bien équipé à cet effet.

\section{RESULTATS}

\section{Données pétrographiques}

Les observations de terrain ont permis de distinguer les formations de socle et les formations volcaniques de couverture. Les formations de socle représentent environ $2 / 5$ de la superficie et sont constituées de roches orthodérivées et des roches franchement métamorphiques.

\section{Les roches orthodérivées ; les orthogneiss}

Les orthogneiss sont des roches dont le caractère métamorphique n'est lié qu'à l'orientation préférentielle de certains minéraux. Ils se subdivisent en orthogneiss à mégacristaux de feldspath et en orthogneiss à grain fin. La texture est granoblastique hétérogranulaire. L'association minérale caractéristique est la suivante: QuartzOrthose-Microcline-Biotite-Amphibole-

Plagioclase-Apatite-Sphène.

\section{Les roches franchement métamorphiques}

Les roches franchement métamorphiques sont représentées par les migmatites, les gneiss, les amphibolites traversés par des filons :

- les migmatites présentent un leucosome, un mélanosome, et un mésosome avec parfois un aspect rubané de type litage compositionnel. La texture est granoblastique. L'association minérale caractéristique est la suivante: Quartz-Orthose-Microcline-Amphibole-

Biotite-Plagioclase-Zircon. Les marques de la déformation sont généralement enregistrées par le quartz en ruban et par la biotite.

- les gneiss présentent une alternance plus ou moins nette de lits clairs millimétriques riches en quartz et feldspath et des lits sombres plurimillimétriques riches en minéraux ferromagnésiens. La texture est granoblastique hétéro-granulaire orientée. L'association minérale est constante: Quartz-Feldspath alcalin-Plagioclase-Hornblende-Biotite qu'accompagnent Apatite-Sphène et minéraux opaques. La déformation est marquée par des sigmoïdes de feldspath et d'amphibole, la biotite, des rubans de quartz orientés dans le plan de la foliation.

- les amphibolites sont des roches sombres à texture granoblastique à grain moyen présentant une orientation préférentielle des minéraux (amphibole, feldspaths et quartz).

- les filons traversent les formations encaissantes (gneiss, migmatites, amphibolites, et orthogneiss). Ce sont des filons aplitiques d'épaisseur centimétriques et des filons pegmatitiques d'épaisseur centimétrique à décamétrique.

$$
\text { Les formations volcaniques }
$$
représentent environ $3 / 5$ des hauts plateaux de 
l'Ouest. On distingue basaltes (sub-aphyriques et porphyriques), phonolites, trachytes, rhyolites, tufs et ignimbrites.

Les basaltes sub-aphyriques sont les plus nombreux. Leur cassure est franche et leur texture microlitique à tendance fluidale. La mésostase représente $85 \%$ du volume de la roche et le verre $15 \%$. Les minéraux constitutifs sont: olivine, pyroxène, plagioclase et opaques. Ces basaltes renferment de nombreuses géodes (pouvant atteindre plusieurs centimètres de diamètre) à cristallisation secondaire de quartz, calcite et zéolite qui se retrouve également dans les fissures et micro-fissures qui parcourent ces laves.

Les basaltes porphyriques ont une cassure de type ovoïde et leur texture est microlitique porphyrique à tendance fluidale. Les phénocristaux dont la taille est comprise entre 1,5 et $6 \mathrm{~mm}$ sont constitués de plagioclase, d'olivine et de pyroxène qui occupent 3 à $25 \%$ du volume de la roche. La mésostase (75 à 97\%) contient le plagioclase, l'olivine, le pyroxène et les minéraux opaques. La cristallisation secondaire n'est pas ici aussi accentuée que dans le type aphyrique.

Les phonolites ont une texture microlitique sub-porphyrique à tendance fluidale. Les phénocristaux représentent 8 à $15 \%$ de la roche et sont formés de feldspath alcalin, plagioclase, clinopyroxène, et parfois amphibole. La mésostase représente 85 à $92 \%$ du volume de la roche et montre dans une matrice dévitrifiée des baguettes de feldspath, de microcristaux de clinopyroxène, de biotite, d'amphibole, et d'oxydes.

Les trachytes ont une texture microlitique porphyrique. Les phénocristaux représentent 20 à $25 \%$ du volume de la roche et sont formés de feldspath, clinopyroxène, et amphibole alors que la mésostase (75 à $80 \%$ du volume total de la roche) est constituée de fins cristaux des mêmes minéraux.

Les tufs ont un aspect pulvérulent et se sont parfois consolidés à chaud pour former les ignimbrites s.s. (Nono et al., 2003). Les ignimbrites ont une texture vitroclastique ; les phénocristaux sont ceux de feldspath alcalin et quartz dans une matrice très dévitrifiée constituée de microflammes, de quartz et de feldspath.
Les rhyolites ont une texture microlitique porphyrique. Les phénocristaux représentent 5 à $10 \%$ du volume de la roche et sont constitués de feldspath alcalin, et quartz. La mésostase représente 90 à $95 \%$ et montre de fins cristaux de feldspath et d'opaques.

\section{Données structurales \\ Analyses linéamentaire et morphostructurale}

Dans le secteur SW des hauts plateaux de l'Ouest-Cameroun, l'analyse morphostructurale montre qu'en général les vallées et les collines sont allongées suivant les mêmes directions à savoir : NE-SW, NW-SE et E-W. Ceci permet de dire que la tectonique a influencé de façon considérable le tracé du réseau hydrographique et le façonnement de l'orographie.

La photo interprétation a permis de mettre en évidence dans ce secteur des hauts plateaux de l'Ouest l'existence des linéaments kilométriques qui correspondent sur le terrain à la rectitude des contours géomorphologiques et à la disposition du réseau hydrographique. La rosace des directions linéamentaires met en évidence trois directions principales (N70$\left.80^{\circ} \mathrm{E}, \quad \mathrm{N} 130-140^{\circ} \mathrm{E}, \quad \mathrm{N} 50-60^{\circ} \mathrm{E}\right)$ et cinq directions secondaires (N100-110 ${ }^{\circ} \mathrm{E}, \mathrm{N} 110$ $120^{\circ} \mathrm{E}, \quad \mathrm{N} 120-130^{\circ} \mathrm{E}, \quad \mathrm{N} 20-40^{\circ} \mathrm{E}$, et $\mathrm{N} 140-$ $\left.150^{\circ} \mathrm{E}\right)$. La rosace de direction de longueur cumulée montre trois directions majeures à savoir $\mathrm{N} 70-80^{\circ} \mathrm{E}, \mathrm{N} 20-40^{\circ} \mathrm{E}, \mathrm{N} 120-140^{\circ} \mathrm{E}$ et deux directions secondaires $\mathrm{N} 40-60^{\circ} \mathrm{E}$, et N140- $160^{\circ} \mathrm{E}$ (Figure 2).

\section{Eléments structuraux (Données de terrain)}

Les éléments structuraux concernent les formations du socle et on peut distinguer : - la foliation qui résulte de l'alternance des lits clairs et des lits sombres dans les formations métamorphiques a une orientation allant de $\mathrm{N} 25^{\circ} \mathrm{E}$ à $\mathrm{N} 75^{\circ} \mathrm{E}$ et de pendage $30-40^{\circ}$ vers le Sud et Sud-Ouest.

- la schistosité a des orientations allant de N-S à $\mathrm{N} 60^{\circ} \mathrm{E}$ avec des pendages variant de 30 à $90^{\circ}$ vers le SSW, SW et ESE.

- les plis reprennent en général la schistosité, leurs axes sont orientés N30-40 ${ }^{\circ} \mathrm{E}, \mathrm{N} 60-70^{\circ} \mathrm{E}$, avec des plongements variant de 20 à $30^{\circ}$ vers le $\mathrm{S}$ et le WSW.

- les diaclases ont deux faisceaux de directions à savoir $\mathrm{N} 20-50^{\circ} \mathrm{E}$ et $\mathrm{N} 80-130^{\circ} \mathrm{E}$ qui s'apparentent bien aux directions des linéaments. 
L'analyse linéamentaire et morphostructurale permet de constater que les accidents tectoniques orientés $\mathrm{N} 70-80^{\circ} \mathrm{E}$,
$\mathrm{N} 20-40^{\circ} \mathrm{E}$ et $\mathrm{N} 150-160^{\circ} \mathrm{E}$ ont été les plus fréquents et les plus intenses. Ces orientations concordent avec les directions des grands

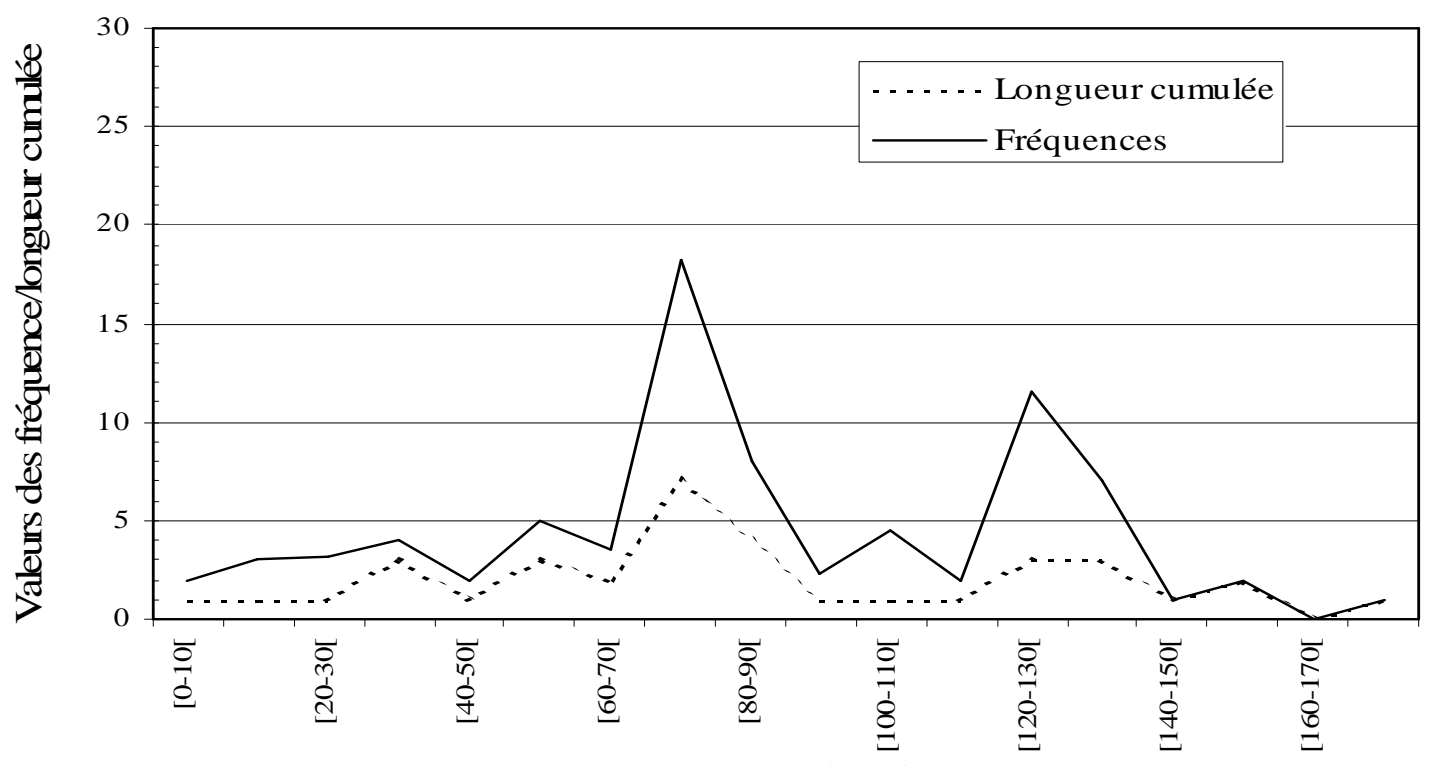

Classes de direction

Figure 2 : Directions structurales majeures dans les Hauts Plateaux de l'Ouest-Cameroun.

Tableau 1 : Données de foration.

\begin{tabular}{|c|c|c|c|c|c|c|c|}
\hline Localité & Arrondissement & Département & $\begin{array}{l}\text { Coordonnées } \\
\text { GPS, Forage }\end{array}$ & Lithologie & $\begin{array}{c}\text { Profondeur } \\
\text { (m) forage }\end{array}$ & $\begin{array}{l}\text { Epaisseur } \\
\text { altérite } \\
(\mathbf{m}) \\
\end{array}$ & $\begin{array}{l}\text { Débit } \\
\left(\mathrm{m}^{3} / \mathbf{h}\right)\end{array}$ \\
\hline Bakouké & Mbouda & Bamboutos & $\begin{array}{l}\text { N: } 05^{\circ} 38,769^{\prime} \\
E: 10^{\circ} 09,879^{\prime}\end{array}$ & Basalte & 51,5 & 23 & 0,9 \\
\hline Poango & Banka & Haut Nkam & $\begin{array}{l}\text { N: } 05^{\circ} 09,443^{\prime} \\
E: 10^{\circ} 12,009^{\prime}\end{array}$ & Basalte & 28,5 & 7 & 0,75 \\
\hline Famgham & Bandja & Haut Nkam & $\begin{array}{l}\text { N: } 05^{\circ} 17,886^{\prime} \\
E: 10^{\circ} 13,942^{\prime}\end{array}$ & Granite & 38 & 10 & 3,5 \\
\hline $\begin{array}{l}\text { Bafou } \\
\text { (Melekouet) }\end{array}$ & Dschang & Menoua & $\begin{array}{l}\text { N: } 05^{\circ} 30,898^{\prime} \\
E: 10^{\circ} 07,203^{\prime}\end{array}$ & Basalte & 81,5 & 14 & 2,4 \\
\hline Nzenla & Dschang & Menoua & $\begin{array}{l}\text { N: } 05^{\circ} 23,902^{\prime} \\
E: 09^{\circ} 58,179^{\prime}\end{array}$ & Granite & 45 & 5 & 0,8 \\
\hline Bamelieu & Nkongni & Menoua & $\begin{array}{l}\mathrm{N}: 05^{\circ} 31,134^{\prime} \\
\mathrm{E}: 10^{\circ} 05,455^{\prime}\end{array}$ & Basalte & 51 & 22 & 2 \\
\hline Fokamezo & Nkongni & Menoua & $\begin{array}{l}N: 05^{\circ} 24,748^{\prime} \\
E: 10^{\circ} 08,108^{\prime}\end{array}$ & Basalte & 47,2 & 30 & 3,3 \\
\hline Bamendou & $\begin{array}{l}\text { Penka } \\
\text { Michel }\end{array}$ & Menoua & $\begin{array}{l}\text { N: } 05^{\circ} 24,779^{\prime} \\
\mathrm{E}: 10^{\circ} 09,299^{\prime}\end{array}$ & Granite & 30 & 25 & 3,6 \\
\hline $\begin{array}{l}\text { Banie- } \\
\text { Baloum }\end{array}$ & $\begin{array}{l}\text { Penka } \\
\text { Michel }\end{array}$ & Menoua & $\begin{array}{l}\text { N: } 05^{\circ} 22,633^{\prime} \\
\text { E: } 10^{\circ} 11,459^{\prime}\end{array}$ & Granite & 61,5 & 25 & 0,7 \\
\hline $\begin{array}{l}\text { Fondjo- } \\
\text { menkouet }\end{array}$ & Bandja & Haut Nkam & $\begin{array}{l}\text { N: } 05^{\circ} 17,530^{\prime} \\
\text { E: } 10^{\circ} 11,855^{\prime}\end{array}$ & $\begin{array}{l}\text { Granito- } \\
\text { gneiss } \\
\text { mylonitise }\end{array}$ & 32 & 20 & 20 \\
\hline
\end{tabular}


accidents régionaux à savoir la Ligne du Cameroun $\left(\mathrm{N} 20-30^{\circ} \mathrm{E}\right)$ et les décrochements dans le domaine centre de la chaîne panafricaine nord équatoriale $\left(\mathrm{N} 30^{\circ} \mathrm{E}\right.$ et N70 ${ }^{\circ}$ E) (Nzenti et al., 1994; Nzenti et Tchoua, 1996). Ces accidents sont des points de vulnérabilité qu'empruntent les eaux au cours de l'altération des roches et sont d'un grand intérêt pour la productivité des aquifères dans les hauts plateaux de l'Ouest-Cameroun (Nono et al., 2004b).

\section{Données géophysiques et résultats de foration}

La prospection géophysique a permis d'obtenir les résistivités apparentes dont les

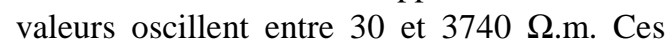
variations des résistivités permettent de subdiviser la région d'étude en quatre couches :

- Une couche moins résistante entre 1 et $25 \mathrm{~m}$ de profondeur ; qui correspondrait à la couche d'altération,

- Une couche résistante entre 25 et $35 \mathrm{~m}$ de profondeur ; qui serait le toit de l'aquifère,

- un terrain conducteur localisé entre 35 et 70 $\mathrm{m}$ de profondeur; qui correspondrait a l'aquifère,

- un terrain compact localisé entre 70 et $120 \mathrm{~m}$ de profondeur; qui correspondrait au mur de l'aquifère.

Les résultats des travaux de foration présentés dans le tableau 1 montrent de façon générale que la couche d'altération évolue jusqu'à une profondeur de $30 \mathrm{~m}$ dans la zone de Dschang (localité de Fokamezo) sur des formations basaltiques. Les forages réalisés dans la région d'étude présentent des débits généralement inférieurs à $8 \mathrm{~m}^{3} / \mathrm{h}$. Néanmoins, on a exceptionnellement obtenu un débit de 20 $\mathrm{m}^{3} / \mathrm{h}$ dans la zone de Bandja dans un forage réalisé sur le socle granito-gneissique bien mylonitisé.

\section{Aperçu minéralogique et géochimique}

Les données minéralogiques et
géochimiques nous permettront de comprendre l'origine des éléments chimiques majeurs dans les eaux souterraines.

Le tableau 2 montre que dans l'ensemble, la teneur en $\mathrm{SiO}_{2}$ varie de 42 à $75 \%$ d'un faciès à un autre, celle en $\mathrm{Al}_{2} \mathrm{O}_{3}$ de 11 à $16 \%$, celle des alcalino-terreux $(\mathrm{FeO}+$
$\mathrm{CaO}+\mathrm{MgO})$ de 35 à $2 \%$, alors que celle des alcalins $\left(\mathrm{Na}_{2} \mathrm{O}+\mathrm{K}_{2} \mathrm{O}\right)$ varie de $\left.4-11 \%\right)$.

Les granitoïdes montrent une évolution typique calco-alcaline marquée par une large gamme de variation dans la composition chimique: $48-76 \% \quad \mathrm{SiO}_{2}, 5-10 \% \mathrm{CaO}, 4-10 \%$ $\left(\mathrm{Na}_{2} \mathrm{O}+\mathrm{K}_{2} \mathrm{O}\right), 0-12 \%\left(\mathrm{Fe}_{2} \mathrm{O}_{3}\right), 0-2 \% \quad \mathrm{TiO}_{2}$. $\mathrm{P}_{2} \mathrm{O}_{5}$ et $\mathrm{MnO}$ ont des valeurs inférieures à $1 \%$ (Talla, 1995; Kwékam, 2005).

Les roches plutoniques ont une tendance alcaline à hyper-alcaline (présence d'aegyrine et d'arfvedsonite) et présentent une variation significative de concentrations en éléments majeurs des dolérites aux granites : $50-72 \% \mathrm{SiO}_{2}, 11-16 \% \mathrm{Al}_{2} \mathrm{O}_{3}, 1-9 \% \mathrm{Fe}_{2} \mathrm{O}_{3}, 4-$ $0 \% \mathrm{MgO}, 9-0 \% \mathrm{CaO}, 5-11 \%\left(\mathrm{Na}_{2} \mathrm{O}+\mathrm{K}_{2} \mathrm{O}\right)$ (Wouatong, 1998).

Les roches volcaniques de couverture qui affleurent largement sur les hauts plateaux de l'Ouest-Cameroun (surtout dans les Monts Bambouto, Bana et Bangou), sont comme les roches plutoniques, alcalines à hyperalcalines, mais avec une affinité transitionnelle aux Monts Bangou et Bana (Fosso et al., 2005; Kuepouo et al., 2006). Elles présentent une large variation de composition minéralogique des roches mafiques aux roches felsiques: olivine + augites + plagioclase (bytownite) dans les basaltes alcalins, puis plagioclase (bytownite, labrador, andésine) + pyroxène (salite, augite) + rares cristaux d'olivine dans les hawaïtes et enfin plagioclase (labrador, andésine, oligoclase, albite) + pyroxène (augite, aegyrine) + k-feldspath (sanidine, anorthose) + rares cristaux d'amphibole et biotite dans les laves felsiques. La distribution des éléments majeurs largement influencée par le fractionnement des minéraux sus-cités montre également une large variation des basaltes aux rhyolites : $42-75 \% \mathrm{SiO}_{2}, 16-11 \%$ $\mathrm{Al}_{2} \mathrm{O}_{3}, 15-1 \% \quad \mathrm{Fe}_{2} \mathrm{O}_{3}, 12-0 \% \mathrm{MgO}, 11-0 \%$ $\mathrm{CaO}, 3-11 \%\left(\mathrm{Na}_{2} \mathrm{O}+\mathrm{K}_{2} \mathrm{O}\right)$ (Marzoli et al., 1999, 2000; Fosso et al., 2005).

Dans l'ensemble, les formations géologiques des hauts plateaux de l'OuestCameroun ont des teneurs en $\mathrm{P}_{2} \mathrm{O}_{5}$ entre $2 \%$ dans les roches basiques à presque $0 \%$ dans les roches acides. Les teneurs en $\mathrm{MnO}$ et $\mathrm{CO}_{2}$ sont faibles $(\leq 1 \%)$, mais $\mathrm{CO}_{2}$ atteint $2 \%$ dans certains trachytes des Monts Bambouto (Nni et Nyobe, 1995).

Les analyses chimiques (Talla, 1995; Kwékam, 2005) montrent que les roches du socle présentent des éléments majeurs 
A. NONO et al. / Int. J. Biol. Chem. Sci. 3(2): 218-239, 2009

Tableau 2: Analyses chimiques des roches représentatives des Hauts Plateaux de l'Ouest-Cameroun: Région de Dschang-Bambouto.

\begin{tabular}{|c|c|c|c|c|c|c|c|c|c|c|c|c|c|c|c|c|}
\hline \multirow{2}{*}{$\begin{array}{l}\text { Roches } \\
\text { Echantillon }\end{array}$} & \multicolumn{2}{|c|}{ Hawaïtes } & \multicolumn{5}{|c|}{ Basaltes } & \multicolumn{2}{|c|}{ Trachyte } & \multicolumn{2}{|c|}{ Rhyolite } & \multicolumn{2}{|c|}{ Orthogneiss } & \multicolumn{3}{|c|}{ Granites } \\
\hline & F55 & F56 & CA54 & CA40 & F54 & F51 & Tap1 & F59 & CA24 & F57 & CA65 & K513 & SAFP & $\mathrm{X} 30$ & GD09 & SAG12 \\
\hline $\mathrm{SiO}_{2} \quad \mathrm{Wt} \%$ & 48,95 & 47,86 & 42,38 & 47,32 & 46,39 & 46,09 & 43,63 & 67,68 & 68,87 & 74,71 & 67,09 & 56,66 & 65,77 & 71,27 & 68,8 & 69,33 \\
\hline $\mathrm{TiO}_{2}$ & 2,89 & 2,92 & 4,24 & 2,87 & 3,64 & 3,60 & 2,86 & 0,29 & 0,86 & 0,21 & 0,36 & 1,86 & 0,47 & 0,37 & 0,46 & 0,54 \\
\hline $\mathrm{Al}_{2} \mathrm{O}_{3}$ & 14,91 & 16,00 & 12,79 & 15,27 & 13,41 & 14,78 & 12,87 & 14,26 & 12,70 & 10,75 & 14,61 & 15,86 & 14,82 & 13,67 & 15,2 & 14,07 \\
\hline $\mathrm{Fe}_{2} \mathrm{O}_{3} *$ & 13,73 & 12,84 & 12,30 & 11,28 & 15,05 & 8,80 & 13,14 & 0,95 & 5,92 & 3,92 & 6,31 & 8,73 & 5,31 & 3,70 & 3,34 & 4,85 \\
\hline $\mathrm{MnO}$ & 0,18 & 0,15 & 0,19 & 0,18 & 0,23 & 0,09 & 0,20 & 0,04 & 0,23 & 0,07 & 0,12 & 0,09 & 0,09 & 0,04 & 0,03 & 0,03 \\
\hline $\mathrm{MgO}$ & 3,49 & 5,84 & 11,54 & 7,23 & 4,61 & 3,20 & 11,41 & 0,02 & 0,35 & 0,06 & 0,05 & 3,02 & 1,46 & 0,34 & 0,57 & 0,51 \\
\hline $\mathrm{CaO}$ & 7,79 & 8,35 & 11,63 & 10,39 & 9,18 & 5,27 & 10,62 & 1,13 & 0,24 & 0,01 & 2,21 & 4,98 & 3,69 & 1,34 & 1,76 & 1,51 \\
\hline $\mathrm{K}_{2} \mathrm{O}$ & 1,88 & 1,19 & 1,30 & 1,19 & 1,01 & 2,67 & 1,06 & 6,09 & 4,90 & 4,63 & 5,43 & 3,89 & 4,76 & 5,22 & 5,45 & 5,52 \\
\hline $\mathrm{P}_{2} \mathrm{O}_{5}$ & 0,85 & 0,76 & 0,94 & 0,49 & 1,79 & 0,43 & 0,72 & 0,04 & 0,05 & 0,02 & 0,10 & 0,73 & 0,14 & 0,12 & 0,18 & 0,17 \\
\hline L.O.I. & 1,86 & 0,36 & 2,18 & 1,43 & 1,10 & 1,19 & 0,41 & 3,84 & 1,07 & 1,49 & 1,47 & & & & & \\
\hline Total & 99,69 & 99,71 & 100,00 & 99,70 & 99,70 & 99,77 & 99,98 & 99,32 & 100,00 & 99,75 & 100,00 & 99,97 & 99,97 & 99,33 & 99,36 & 99,67 \\
\hline $\mathrm{K}_{2} \mathrm{O} / \mathrm{Na}_{2} \mathrm{O}$ & 0,59 & 0,35 & 0,56 & 0,34 & 0,30 & 0,70 & 0,34 & 1,8 & 0,80 & 1,10 & 1,46 & 1,08 & 1,54 & 1,63 & 1,6 & 1,76 \\
\hline $\mathrm{K}_{2} \mathrm{O}+\mathrm{NaO}_{2}$ & 5,04 & 4,61 & 4,99 & 4,67 & 4,30 & 6,16 & 4,12 & 9,30 & 10,79 & 8,51 & 9,15 & 7,49 & 7,84 & 8,47 & 8,47 & 8,65 \\
\hline $\mathrm{Ba} \mathrm{ppm}$ & 612 & 409 & 655 & 387 & 332 & 251 & 574 & 1561 & 50 & 40,2 & 1091 & 1815 & 2239 & 580 & 1304 & 765 \\
\hline $\mathrm{Cr}$ & 17,6 & 78,6 & 356 & 338 & 15,4 & 24,5 & 310 & $\operatorname{tr}$ & & 8,26 & & 20 & $<20$ & 33 & 13 & 49 \\
\hline $\mathrm{Ga}$ & 28,2 & 24,9 & & & 24,6 & 24,5 & 16 & 32,4 & & 3,33 & & 21 & 14 & 17 & 23 & 19 \\
\hline $\mathrm{Hf}$ & 8,24 & 5,35 & & & 5,34 & 4,4 & 4,8 & 17,5 & & 22,2 & & & & & & \\
\hline $\mathrm{Ni}$ & 25,9 & 71,7 & 183 & 189 & 16,1 & 19,1 & 220 & 1,91 & & 11,5 & & 30 & $<20$ & 4 & 5 & $<3$ \\
\hline $\mathrm{Rb}$ & 50,7 & 29,1 & 32 & 32 & 23 & 13,1 & 43 & 152 & 148 & 137 & 136 & 152 & 65 & 295 & 195 & 211 \\
\hline $\mathrm{Sr}$ & 545 & 528 & 1643 & 610 & 537 & 529 & 821 & 136 & 9 & 2,42 & 102 & 682 & 407 & 138 & 279 & 186 \\
\hline $\mathrm{Ta}$ & 2,71 & 2,11 & & & 2,24 & 1,79 & 4,3 & 17,5 & & 7,86 & & & & & & \\
\hline Th & 5,36 & 3,29 & & & 3,47 & 2,30 & 4,7 & 17,1 & & 18,4 & & & & 48 & & 24 \\
\hline V & 208 & 215 & & & 229 & 279 & 239 & 0,42 & & 0,01 & & 90 & 54 & 27 & 19 & 37 \\
\hline Y & 42 & 28,5 & 34 & 33 & 42,8 & 27,9 & 27 & 67,2 & 80 & 71 & 97 & 23 & 29 & 9 & 10 & 7 \\
\hline $\mathrm{Zn}$ & 156 & 1,30 & & & 152 & 129 & 90 & 131 & & 144 & & 120 & 50 & 51 & 83 & 51 \\
\hline $\mathrm{Zr}$ & 348 & 237 & 151 & 243 & 225 & 209 & 201 & 686 & 1573 & 824 & 1208 & 646 & 112 & 217 & 336 & 283 \\
\hline
\end{tabular}

227 
A. NONO et al. / Int. J. Biol. Chem. Sci. 3(2): 218-239, 2009

\begin{tabular}{|c|c|c|c|c|c|c|c|c|c|c|c|c|c|}
\hline La ppm & 49,87 & 32,81 & 49,85 & 37,35 & 39,45 & 24,59 & 47,4 & 104,9 & 157,9 & 102,6 & 131 & 112 & 112 \\
\hline $\mathrm{Ce}$ & 105,7 & 71,6 & 105,5 & 76,25 & 91,28 & 54,05 & 93,3 & 198 & 324,5 & 207,5 & 257 & 175 & 151 \\
\hline $\operatorname{Pr}$ & 13,07 & 9,05 & & & 11,91 & 7,07 & 10,1 & 23,04 & & 23,32 & & & \\
\hline $\mathrm{Nd}$ & 55,9 & 39,82 & 57,21 & 37,95 & 54,67 & 31,93 & 42,8 & 90,1 & 130,1 & 92,76 & 128 & 59 & $<4$ \\
\hline $\mathrm{Sm}$ & 11,87 & 8,67 & 11,99 & 8,10 & 13,7 & 7,75 & 9 & 17,72 & 24,26 & 18,46 & & & \\
\hline $\mathrm{Gd}$ & 10,15 & 7,36 & 8,78 & 6,70 & 11,3 & 6,74 & 7,4 & 14,24 & 17,90 & 15,17 & & & \\
\hline $\mathrm{Tb}$ & 1,58 & 1,1 & & & 1,63 & 1,04 & 1,1 & 2,18 & & 2,39 & & & \\
\hline Dy & 8,43 & 5,79 & 5,87 & 5,44 & 8,88 & 5,56 & 5,6 & 12,4 & 14,80 & 14,03 & & & \\
\hline Но & 1,75 & 1,18 & & & 1,68 & 1,13 & 0,9 & 2,66 & & 2,93 & & & \\
\hline Er & 3,82 & 2,46 & 2,47 & 2,47 & 3,71 & 2,50 & 2,4 & 6,15 & 7,57 & 6,8 & & & \\
\hline $\mathrm{Tm}$ & 0,54 & 0,49 & & & 0,49 & 0,35 & 0,32 & 0,93 & & 1 & & & \\
\hline $\mathrm{Yb}$ & 3,48 & 2,16 & 2,01 & 2,05 & 3,03 & 2,11 & 1,8 & 5,92 & 7,71 & 6,42 & & & \\
\hline $\mathrm{Lu}$ & 0,46 & 0,30 & 0,26 & 0,30 & 0,42 & 0,30 & 0,25 & 0,90 & 1,20 & 0,99 & & & \\
\hline
\end{tabular}
Région de Batié; X30 et SAG12 (Granites); Talla, 1995, Région de Bangou-Bandjoun; F54 et F51 (basaltes), F55 ET F56 (Hawaïtes), F59 (Trachyte) et F57 (Rhyolite), Fosso, 2005 , CA65 (Rhyolite); Marzoli et al., 1999. $\operatorname{tr}$ : traces.

Tableau 3 : Résultats d'analyses physico-chimiques des eaux de sources dans les hauts plateaux de l'Ouest. (Niveau d'aménagement: SA: Source aménagée; SNA: Source non aménagée; SSA: Source Sommairement aménagée).

\begin{tabular}{|c|c|c|c|c|c|c|c|c|c|c|c|c|c|c|}
\hline Ech & Niv Am & Faciès & T Lab. & pH & $\begin{array}{l}\text { Cond. } \\
\mu \mathrm{S} / \mathrm{cm}\end{array}$ & $\begin{array}{l}\text { TDS } \\
\mathrm{mg} / \mathrm{l} \\
\end{array}$ & $\begin{array}{l}\mathrm{Na}^{+} \\
\mathrm{mg} / \mathrm{l}\end{array}$ & $\begin{array}{c}\mathrm{K}^{+} \\
\mathrm{mg} / \mathrm{l} \\
\end{array}$ & $\begin{array}{l}\mathrm{Ca}^{++} \\
\mathrm{mg} / \mathrm{l}\end{array}$ & $\begin{array}{c}\mathrm{Mg}^{++} \\
\mathrm{mg} / \mathrm{l} \\
\end{array}$ & $\begin{array}{c}\mathrm{Cl}^{-} \\
\mathrm{mg} / \mathrm{l} \\
\end{array}$ & $\begin{array}{c}\mathrm{HCO}_{3}^{-} \\
\mathrm{mg} / \mathrm{l}\end{array}$ & $\begin{array}{l}\mathrm{SiO}_{2} \\
\mathrm{mg} / \mathrm{l} \\
\end{array}$ & $\begin{array}{l}\mathrm{Fe}^{3+} \\
\mathrm{mg} / \mathrm{l}\end{array}$ \\
\hline $\begin{array}{l}\text { Normes } \\
\text { O.M.S. }\end{array}$ & IVIV Am & F actes & $25^{\circ}$ in situ & 6,5 à 8,5 & $\begin{array}{l}2000 \\
\max \end{array}$ & $\begin{array}{l}1245 \\
\max \end{array}$ & $\begin{array}{l}200 \\
\max \end{array}$ & $\begin{array}{l}290 \\
\max \end{array}$ & $\begin{array}{l}200 \\
\max \end{array}$ & $\begin{array}{l}150 \\
\max \end{array}$ & $\begin{array}{l}250 \\
\max \end{array}$ & $\begin{array}{l}150 \\
\max \end{array}$ & Abscence & $\begin{array}{c}0,3 \\
\max \end{array}$ \\
\hline S1 & SA & $\beta$ ap & 28,5 & 6,5 & 37,46 & 59,88 & 2,34 & 1,31 & 0,4 & 2,25 & 14 & 24,4 & 14,90 & 0,28 \\
\hline $\mathrm{S} 2$ & SA & $\beta$ ap & 28,5 & 6,5 & 138,15 & 238,27 & 4,28 & 1,09 & 8,88 & 2,25 & 16,8 & 92,72 & 112 & 0,25 \\
\hline S3 & SA & $\beta$ ap & 29 & 5,02 & 18,62 & 49,24 & 0,99 & 0,01 & 0,24 & 1,65 & 16,8 & 12,2 & 17,1 & 0,25 \\
\hline S4 & SNA & $\beta$ ap & 28,5 & 5,27 & 22,57 & 41,48 & 1,40 & 0,56 & 0,64 & 0,32 & 1,61 & 17,08 & 16,62 & 3,25 \\
\hline S5 & SNA & $\beta$ ap & 29 & 5,17 & 24,10 & 82,45 & 11,53 & 0,03 & 0,24 & 2,02 & 18,46 & 17,08 & 32,98 & 0,11 \\
\hline S6 & SNA & $\beta$ ap & 28,5 & 5,35 & 16,99 & 49.85 & 0,81 & 0,03 & 0,64 & 1,3 & 19,88 & 14,64 & 12,03 & 0,52 \\
\hline
\end{tabular}


A. NONO et al. / Int. J. Biol. Chem. Sci. 3(2): 218-239, 2009

\begin{tabular}{|c|c|c|c|c|c|c|c|c|c|c|c|c|c|c|}
\hline S7 & SNA & $\beta$ ap & I & 6 & I & 132,14 & 2,2 & 0,14 & 3,61 & 2,92 & 42,55 & 61,01 & 19,7 & $<0,01$ \\
\hline S8 & SNA & $\beta$ ap & I & 6 & I & 118,41 & 0,95 & 0,14 & 3,21 & 2,43 & 38,37 & 61,01 & 12,29 & $<0,01$ \\
\hline S9 & SNA & $\beta$ ap & I & 6,5 & 1 & 132,14 & 2,2 & 0,14 & 3,61 & 2,92 & 42,55 & 61,01 & 19,7 & $<0,01$ \\
\hline S10 & SNA & $\beta$ ap & I & 6,5 & I & 120,26 & 2,4 & 0,14 & 2,81 & 2,43 & 35,46 & 61,01 & 16 & $<0,01$ \\
\hline S11 & SNA & $\beta$ ap & I & 6 & I & 97,7 & $2 ; 20$ & 0,14 & 4,01 & 2,43 & 28,37 & 48,41 & 12,13 & $<0,01$ \\
\hline $\mathrm{S} 12$ & SSA & $\beta$ ap & 28,5 & 4,93 & 34,9 & 87,66 & 4,6 & 1,95 & 1,20 & 0,72 & 18,10 & 2,72 & 58,35 & 0,028 \\
\hline S13 & SSA & $\beta$ ap & 28,5 & 4,86 & 28,00 & 57,8 & 1,63 & 7,82 & 3,56 & 0,60 & 17,04 & 2,32 & 23,18 & 1,65 \\
\hline S14 & SA & $\beta$ ap & 29 & 4,78 & 14,48 & 79,17 & 1,12 & 0,31 & 1,16 & 0,29 & 51,12 & 1,54 & 23,55 & 0,08 \\
\hline S15 & SA & $\beta$ ap & 29,5 & 5,01 & 24,62 & 133,02 & 1,70 & 0,15 & 1,20 & 1,36 & 25,5 & 62,32 & 40,74 & 0,056 \\
\hline S16 & SSA & $\beta$ por & 28,5 & 5,04 & 62,75 & 104,39 & 5,06 & 2,15 & 2,48 & 2,74 & 24,14 & 6,18 & 59,85 & 1,79 \\
\hline S17 & SA & $\beta$ por & I & 6 & I & 105,22 & 0,95 & 0,14 & 3,61 & 0,73 & 56,74 & 36,61 & 6,43 & $<0,01$ \\
\hline S18 & SA & $\beta$ por & I & 6 & I & 113 & 1,78 & 0,48 & 2,81 & 1,95 & 49,64 & 48,81 & 7,42 & 0,11 \\
\hline S19 & SNA & $\beta$ por & I & 6 & I & 76,18 & 0,74 & 0,14 & 2,00 & 0,97 & 28,37 & 36,61 & 7,34 & $<0,01$ \\
\hline S20 & SA & $\beta$ por & 28,5 & 4,96 & 15,57 & 31,47 & 1,44 & 1,09 & 0,32 & 0,46 & 17,04 & 7,32 & 3,80 & 0,00 \\
\hline $\mathrm{S} 21$ & SSA & $\beta$ por & 28,5 & 5,28 & 22,52 & 61,35 & 1,71 & 0,10 & 1,2 & 1,63 & 19,6 & 14,6 & 20,3 & 2,21 \\
\hline S22 & SA & $\beta$ por & 29 & 5,93 & 19,15 & 44,45 & 0,72 & 0,94 & 0,48 & 1,15 & 21 & 7,32 & 12,7 & 0,14 \\
\hline $\mathrm{S} 23$ & SSA & $\beta$ por & 28,5 & 5,50 & 14,97 & 64,68 & 0,92 & 0,63 & 0,52 & 11,11 & 11,20 & 7,32 & 31,50 & 1,48 \\
\hline S24 & SA & $\beta$ por & 28,5 & 5,52 & 21,95 & 56,94 & 0,95 & 0,25 & 0,52 & 1,6 & 15,40 & 12,20 & 24,2 & 1,82 \\
\hline S25 & SA & $\beta$ por & 28,5 & 5,54 & 21,37 & 65,04 & 1,26 & 0,86 & 0,64 & 1,6 & 19,6 & 14,64 & 25,10 & 1,34 \\
\hline S26 & SA & $\beta$ por & 28 & 5,33 & 16,87 & 54,60 & 1,13 & 2,98 & 0,36 & 1,05 & 19,6 & 7,32 & 20,4 & 1,76 \\
\hline S27 & SSA & $\beta$ por & 28 & 5,15 & 24,29 & 61,63 & 1,13 & 0,41 & 0,24 & 2,18 & 18,20 & 17,08 & 20,60 & 1,79 \\
\hline S28 & SSA & phon & 28,5 & 4,31 & 20,78 & 53,96 & 1,31 & 0,3 & 0,4 & 1,51 & 11,20 & 12,20 & 25,60 & 1,44 \\
\hline S29 & SA & phon & 28,5 & 4,20 & 17,98 & 59,23 & 1,17 & 0,71 & 0,60 & 1,20 & 15,40 & 9,76 & 30,20 & 0,25 \\
\hline S30 & SSA & orthg & 29 & 4,99 & 19,75 & 55,71 & 1,53 & 1,92 & 0,4 & 0,77 & 21,3 & 0,76 & 28,89 & 0,14 \\
\hline S31 & SNA & orthg & 28,5 & 5,03 & 21,76 & 69,57 & 1,58 & 1,77 & 0,2 & 0,89 & 19,88 & 12,20 & 32,92 & 0,13 \\
\hline S32 & SNA & orthg & 28,5 & 5,21 & 31,98 & 99,66 & 2,89 & 1,47 & 0,2 & 2,18 & 24,14 & 21,96 & 43,12 & 3,70 \\
\hline S33 & SNA & orthg & 28,5 & 5,26 & 31,10 & 76,62 & 2,70 & 1,47 & 1,16 & 1,66 & 19,88 & 19,52 & 27,25 & 2,98 \\
\hline
\end{tabular}


A. NONO et al. / Int. J. Biol. Chem. Sci. 3(2): 218-239, 2009

\begin{tabular}{|c|c|c|c|c|c|c|c|c|c|c|c|c|c|c|}
\hline S34 & SNA & orthg & 29 & 5,33 & 23,10 & 68 & 1,44 & 0,63 & 0,56 & 1,82 & 18,46 & 17,08 & 24,74 & 3,27 \\
\hline S35 & SA & orthg & 28,5 & 5,15 & 89,30 & 78,13 & 7,04 & 2,00 & 3,16 & 3,58 & 28,30 & 18,08 & 15,91 & 0,06 \\
\hline S36 & SNA & orthg & I & 6,5 & I & 138,2 & 3,24 & 1,18 & 5,21 & 4,38 & 28,37 & 73,21 & 22,6 & $<0,01$ \\
\hline S37 & SSA & orthg & I & 6,5 & I & 138,95 & 4,07 & 0,14 & 8,42 & 5,11 & 28,37 & 73,21 & 19,62 & $<0,01$ \\
\hline S38 & SSA & orthg & 28,5 & 4,91 & 13,13 & 44,7 & 1,15 & 1,36 & 1,20 & 0,48 & 15,62 & 1,16 & 22,89 & 0,84 \\
\hline S39 & SNA & orthg & 28,5 & 5,10 & 19,35 & 53,02 & 1,15 & 0,93 & 1,28 & 0,63 & 24,14 & 1,16 & 15,50 & 0,42 \\
\hline S40 & SNA & orthg & 28,5 & 5,29 & 39,55 & 87,7 & 3,68 & 1,75 & 2,96 & 3,13 & 17,04 & 3,48 & 53,42 & 2,24 \\
\hline S41 & SNA & orthg & 28,5 & 5,28 & 42,13 & 135,24 & 5,29 & 2,15 & 1,20 & 1,43 & 14,2 & 4,25 & 105,83 & 0,89 \\
\hline S42 & SNA & orthg & 28,5 & 5,39 & 48,00 & 108,59 & 4,14 & 1,29 & 3,12 & 2,25 & 25,56 & 4,25 & 63,28 & 4,70 \\
\hline S43 & SNA & orthg & 28,5 & 5,41 & 31,98 & 67,57 & 2,64 & 0,62 & 1,56 & 1,87 & 12,78 & 2,32 & 41,16 & 4,62 \\
\hline S44 & SNA & orthg & 29 & 5,46 & 31,72 & 73,08 & 1,70 & 0,15 & 1,20 & 1,36 & 25,56 & 2,32 & 40,74 & 0,05 \\
\hline S45 & SSA & gniess & I & 6,5 & I & 121,41 & 2,82 & 0,48 & 5,61 & 3,89 & 21,28 & 61,01 & 26,31 & $<0,01$ \\
\hline S46 & SSA & gniess & 28,5 & 5,33 & 31,95 & 63,85 & 2,57 & 1,17 & 2,08 & 1,46 & 17,04 & 21,96 & 16,07 & 1,50 \\
\hline S47 & SSA & $\beta$ ap & 23,1 & 6,5 & 48,9 & 34,3 & 2,8 & 1,5 & 9 & 4 & 0,1 & 10,9 & I & 1 \\
\hline S48 & SSA & $\beta$ ap & 22,7 & 6,5 & 55,7 & 32,6 & 3 & 1,5 & 10 & 5 & 0,1 & 7,02 & I & 0.9 \\
\hline S49 & SNA & $\beta$ ap & 18,2 & 5,8 & 73,8 & 37,3 & 3 & 1,5 & 11 & 2 & 6,5 & 6,1 & I & 0.1 \\
\hline S50 & SSA & $\beta$ ap & 17,3 & 5,3 & 71,3 & 20,1 & 1,1 & 0,7 & 6 & 1 & 0,1 & 6,1 & I & 0.1 \\
\hline S51 & SSA & $\beta$ ap & 19 & 6,5 & 41 & 41 & 1 & 2 & 10 & 1 & 0,1 & 21 & I & 0.1 \\
\hline S52 & SNA & granite & 20,5 & 6,3 & 68,5 & 40,7 & 1,6 & 2,5 & 9 & 1 & 0,1 & 21,4 & I & 0.1 \\
\hline S53 & SNA & granite & 19,8 & 6,4 & 54,9 & 41,1 & 1,3 & 2,25 & 9,5 & 1,5 & 0,1 & 21,4 & I & 0.1 \\
\hline S54 & SSA & trachyte & 22 & 6,6 & 28 & 35,2 & 3 & 3 & 0,1 & 0,1 & 0,1 & 24 & 11 & 0.02 \\
\hline S55 & SSA & trachyte & 23 & 6,5 & 25 & 34,4 & 3 & 2 & 0,2 & 0,1 & 0,1 & 24 & 18 & 0.02 \\
\hline S56 & SNA & ignim & 23 & 6,9 & 17 & 22,9 & 1 & 1 & 0,3 & 0,5 & 0,1 & 15 & 20 & 0.02 \\
\hline S57 & SSA & $\beta$ ap & 22 & 6,2 & 19 & 27,7 & 2 & 0,1 & 0,3 & 0,4 & 0,1 & 20 & 9 & 0.02 \\
\hline S58 & SNA & rhyolite & 21 & 6,3 & 20 & 24,6 & 2 & 2 & 0,3 & 0,2 & 0,1 & 15 & 14 & 0.02 \\
\hline
\end{tabular}

$\beta$ ap $=$ basalte aphyrique, $\beta$ por $=$ basalte porphyrique, phon $=$ phonolite, orthg $=$ orthogneiss, ignim $=$ ignimbrite. 
Tableau 4: Résultats d'analyses physico-chimiques des eaux de forages dans les hauts plateaux de l'Ouest-Cameroun.

\begin{tabular}{|c|c|c|c|c|c|c|c|c|c|c|c|c|c|}
\hline Echantillons & lithologie & $\mathbf{T}^{\circ} \mathbf{C}$ & pH & $\begin{array}{c}\text { Cond } \\
(\mu \mathrm{S} / \mathrm{cm})\end{array}$ & $\begin{array}{l}\text { TDS } \\
\mathrm{mg} / \mathrm{l}\end{array}$ & $\begin{array}{l}\mathrm{Na}+ \\
\mathrm{mg} / \mathrm{l}\end{array}$ & $\underset{\mathrm{mg} / \mathrm{l}}{\mathrm{K}}$ & $\begin{array}{c}\mathrm{Ca} 2+ \\
\mathrm{mg} / \mathrm{l}\end{array}$ & $\underset{\mathrm{mg} / \mathrm{l}}{\mathrm{Mg}+}$ & $\begin{array}{c}\text { Cl- } \\
\text { mg/l }\end{array}$ & $\begin{array}{c}\mathrm{HCO3}- \\
\mathrm{mg} / \mathrm{l}\end{array}$ & $\begin{array}{c}\mathrm{SiO2} \\
\mathrm{mg} / \mathrm{l}\end{array}$ & $\begin{array}{l}\mathrm{Fe} 3+ \\
\mathrm{mg} / \mathrm{l}\end{array}$ \\
\hline BAMENDOU & Granite & 23,1 & 5,9 & 42,5 & 50 & 0,4 & 0,1 & 1 & 0,25 & 0,3 & 21 & nd & 0,63 \\
\hline $\begin{array}{l}\text { BANI- } \\
\text { BALOUM }\end{array}$ & Granite & 22,9 & 5,7 & 35,8 & 85 & 0,4 & 0,1 & 1 & 0,19 & 0,1 & 20 & nd & 0,02 \\
\hline NZENLA & Granite & 23 & 5,6 & 172,3 & 141 & 1,7 & 0,4 & 5,1 & 1,53 & 2 & 130 & nd & 0,08 \\
\hline BAMELIEU & Basalte & 23 & 6,01 & 118,5 & 101 & 1,1 & 0,3 & 3 & 0,96 & 1,5 & 88 & nd & 0,2 \\
\hline FOKAMEZO & Basalte & 23,1 & 5,9 & 114,3 & 208 & 1,8 & 0,2 & 3 & 0,76 & 1,3 & 75 & nd & 0,3 \\
\hline POANGO & Basalte & 23 & 5,8 & 124,8 & 226 & 0,3 & 0,1 & 0,7 & 0,12 & 0,1 & 10 & nd & 1,01 \\
\hline MELEKOUET & Basalte & 23,1 & 5,49 & 340 & 418 & 1,4 & 0,5 & 3 & 0,68 & 1,8 & 100 & nd & 0,81 \\
\hline BOKOTIO & Basalte & 23,5 & 4,72 & 480 & 587 & 1,8 & 0,5 & 18,7 & 6,45 & 11,4 & 170 & nd & 0,16 \\
\hline FAMGHAM & Granite & 24,9 & 6,18 & 280 & 143 & 1 & 0,2 & 12,8 & 3,35 & 5,5 & 110 & nd & 0,36 \\
\hline $\begin{array}{l}\text { FONDJO- } \\
\text { MENKOUET }\end{array}$ & $\begin{array}{c}\text { Granito- } \\
\text { gneiss }\end{array}$ & 24,9 & 6,07 & 347 & 235 & 1,1 & 0,3 & 15,7 & 4,53 & 7,2 & 120 & nd & 0,04 \\
\hline
\end{tabular}

Cond= conductivité, TDS $=$ solides totaux dissous, $\mathrm{nd}=$ non déterminé.

suivants, par ordre de dominance décroissante : $\mathrm{SiO}_{2}, \mathrm{Al}_{2} \mathrm{O}_{3}, \mathrm{Na}_{2} \mathrm{O}, \mathrm{Fe}_{2} \mathrm{O}_{3}$ et $\mathrm{K}_{2} \mathrm{O}$. Ces roches ont des teneurs en silice supérieures à $50 \%$. Le rapport $\mathrm{MgO} / \mathrm{CaO}<1$ traduit une abondance en $\mathrm{Ca}$ et concorde avec le fractionnement des minéraux calciques. Le rapport $(\mathrm{MgO}+\mathrm{CaO}) /\left(\mathrm{Na}_{2} \mathrm{O}+\mathrm{K}_{2} \mathrm{O}\right)$ dans les roches franchement métamorphiques, est supérieur à 1 , ce qui justifie la présence des minéraux ferromagnésiens (biotites), alors que dans les orthogneiss, ce rapport est inférieur ou égal à 1, ce qui concorde avec la prédominance dans ces formations des minéraux sodi-potassiques sur les minéraux ferromagnésiens.

En ce qui concerne les roches volcaniques, les analyses chimiques montrent que les formations basaltiques sont riches en $\mathrm{Mg}, \mathrm{Ca}, \mathrm{Fe}$, et moins riches en $\mathrm{Na}, \mathrm{K}$. alors que les formations acides ont des teneurs en $\mathrm{SiO}_{2}$ plus élevées, ainsi que celles en $\mathrm{K}_{2} \mathrm{O}$ et $\mathrm{Na}_{2} \mathrm{O}$ pour des teneurs en $\mathrm{MgO}$ et $\mathrm{CaO}$ très faibles.

\section{Etude hydrochimique des eaux souterraines \\ Elle porte sur les eaux de 58 sources} (S1 à S58) et 10 forages. Ces sources sont classées suivant le niveau d'aménagement à savoir les sources aménagées (SA), les sources sommairement aménagées (SSA) et les sources non aménagées (SNA). Ainsi 20 sources sur socle (15 sur orthogneiss, 2 sur gneiss, 3 sur granite) et 38 sources sur couverture volcanique (32 sur basaltes, 2 sur phonolites, 2 sur trachytes, 1 sur ignimbrite, 1 sur rhyolite) pour lesquels 17 aménagées, 18 sommairement aménagées et 23 non aménagées ont été analysées (Tableau 3).

- Les analyses chimiques des eaux de sources et de forages étudiées représentées respectivement dans les tableaux 3 et 4 , ont été reportées dans le diagramme de Piper (1944), il en ressort quatre faciès chimiques (Figure 3):

- Le faciès des eaux chlorurées calciques et magnésiennes, $57 \%$ (soit 33 sources) ;

- le faciès des eaux bicarbonatées calciques et magnésiennes, 24\% (soit 14 sources) ;

- le faciès des eaux chlorurées calciques et potassiques, $9 \%$ (soit 5 sources).

- le faciès des eaux bicarbonatées sodiques et potassiques, $10 \%$ (soit 6 sources).

Globalement, les eaux sources ont une tendance chlorurée (soit 66\%), et une tendance calcique et magnésienne (soit $81 \%$ ), comparées aux sources thermominérales de la Ligne du Cameroun (Le Maréchal, 1974) qui sont plutôt bicarbonatées, sodiques et potassiques.

Les eaux des forages quant à elles présentent toutes un faciès bicarbonaté calcique et magnésien. 
Les eaux des sources et des forages analysées sont en général faiblement minéralisées $\quad(\leq 587 \mathrm{mg} / \mathrm{l})$; les plus minéralisées ont les valeurs de conductivité les plus élevées. La minéralisation semble plus ou moins liée aux faciès pétrographiques, puisque des trente deux sources les plus minéralisées $(61,63$ à 238,27 mg/l), dix-huit se trouvent dans les basaltes (10 sur basalte aphyrique et 8 sur basalte porphyrique), quatorze dans le socle (12 sur orthogneiss et 2 sur gneiss). Cependant, les moins minéralisées $(20,1$ à $59,88 \mathrm{mg} / \mathrm{l})$ se trouvent indifféremment dans les orthogneiss, les phonolites et les basaltes aphyriques ou porphyriques. La source la plus minéralisée $(238,27 \mathrm{mg} / \mathrm{l})$ se trouve sur basalte aphyrique, alors que la moins minéralisée $(31,47 \mathrm{mg} / \mathrm{l})$ se trouve sur basalte porphyrique.

Le forage le plus minéralisé $(587 \mathrm{mg} / \mathrm{l})$ a été réalisé sur les formations basaltiques, tandis que le moins minéralisé se trouve sur le socle (granite). Les eaux des forages sont dans l'ensemble plus minéralisées et présentent les valeurs de conductivité les plus élevées.

Toutes les concentrations des éléments chimiques sont conformes aux normes O.M.S., à l'exception de la silice présente dans toutes les sources et du fer dont la teneur est supérieure à $0,3 \mathrm{mg} / \mathrm{l}$ dans 5 sources (4 dans les orthogneiss et 1 dans le basalte aphyrique).

\section{DISCUSSION}

Dans notre secteur comme sur l'ensemble des Hauts plateaux, les formations métamorphiques sont subordonnées aux formations volcaniques (2/5 de la superficie contre 3/5) alors que sur l'ensemble du territoire camerounais les formations métamorphiques couvrent les $2 / 3$ de la surface. Ces formations volcaniques par leur relief plus jeune et plus agressif influencent largement les caractéristiques hydrologiques et hydro-géologiques; accélération du ruissellement, limitation des infiltrations sans oublier les faibles minéralisations des sources (au plus $268 \mathrm{mg} / \mathrm{l}$ ) dues au temps de séjour plus court dans les roches comparée à la situation sur le plateau de l'Adamaoua (jusqu'à 300 mg/l) (Nono and Likeng, 2008).

La fréquence des linéaments et leur importance en longueur cumulée se rapprochent des directions majeures $(\mathrm{N}-\mathrm{S}$,
$\mathrm{N} 70^{\circ} \mathrm{E}, \quad \mathrm{E}-\mathrm{W}$ et $\left.\mathrm{N} 135^{\circ} \mathrm{E}\right)$ proposées par Moreau et al. (1987) pour expliquer la tectonique de la Ligne du Cameroun et de l'Adamaoua. L'analyse linéamentaire et morphostructurale permet de constater que les accidents tectoniques orientés $\mathrm{N} 70-80^{\circ} \mathrm{E}$, $\mathrm{N} 20-40^{\circ} \mathrm{E}$ et $\mathrm{N} 150-160^{\circ} \mathrm{E}$ ont été les plus fréquents et les plus intenses. Ces orientations concordent avec les directions des grands accidents régionaux à savoir la Ligne du Cameroun $\left(\mathrm{N} 20-30^{\circ} \mathrm{E}\right)$ et les décrochements dans le domaine centre de la chaîne panafricaine nord équatoriale $\left(\mathrm{N} 30^{\circ} \mathrm{E}\right.$ et N70 ${ }^{\circ}$ E) (Nzenti et al., 1994; Nzenti et Tchoua, 1996). Ces accidents sont des points de vulnérabilité qu'empruntent les eaux au cours de l'altération des roches qui oriente la formation des reliefs et sont d'un grand intérêt pour la productivité des aquifères dans les hauts plateaux de l'Ouest-Cameroun (Nono et al., 2004b).

Les données de foration montrent que les débits les plus élevés sont obtenus dans les mylonites (roches les plus fracturées), mais aussi dans les formations volcaniques tufacées (Nono et al., 2006) ; ces résultats ressortent de façon précise l'influence des fractures et de la lithologie sur la dynamique des eaux souterraines.

De l'esquisse géochimique et minéralogique, il apparaît que dans les hauts plateaux de l'Ouest, les éléments chimiques les plus dominants dans les roches sont: $\mathrm{SiO}_{2}$, $\mathrm{Al}_{2} \mathrm{O}_{3}, \mathrm{CaO}, \mathrm{MgO}, \mathrm{Fe}_{2} \mathrm{O}_{3}, \mathrm{Na}_{2} \mathrm{O}$ et $\mathrm{K}_{2} \mathrm{O}$; ces éléments sont contenus dans les minéraux ferromagnésiens (olivine, pyroxène, biotite) et des minéraux blancs (plagioclase, sanidine, anorthose, orthose).

Les diagrammes d'équilibre ont permis de mettre en évidence un éloignement des sources étudiées de la courbe d'équilibre de Werner et Giggenbach (1988), un rapprochement de la chimie des sources (rapports cationiques) de celle de la roche hôte (source des ions) et un état d'équilibre entre les eaux de sources et les minéraux secondaires (ou d'altération) caractéristiques des zones tropicales (kaolinite plus abondant, montmorillonite, Ca zéolite rare) (Figures 3 et 4).

Le déséquilibre entre les cations majeurs des sources et ceux de la roche saine montre que la minéralisation se poursuit et l'état d'équilibre entre sources et minéraux 


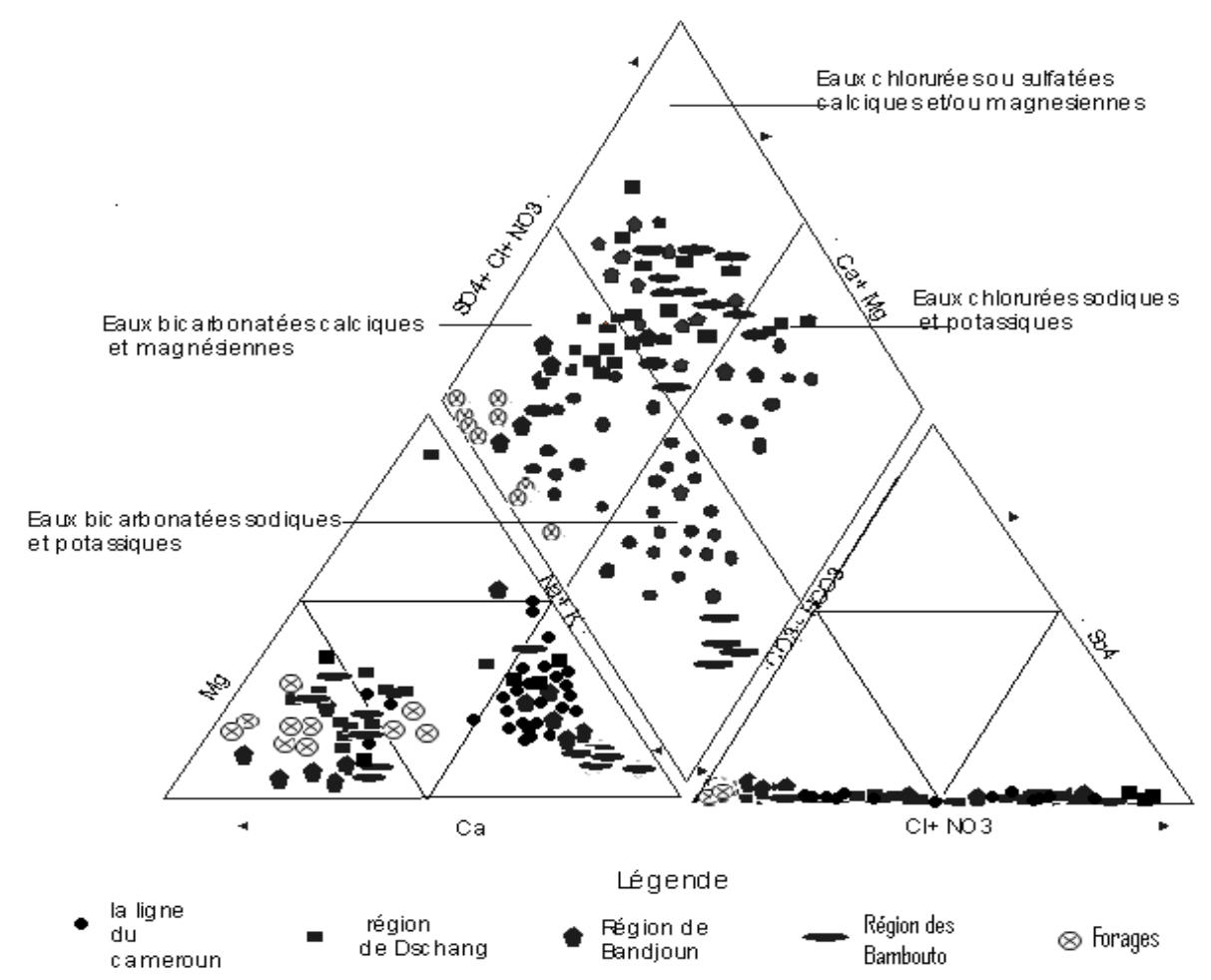

Figure 3: Faciès chimiques des eaux du secteur SW des hauts plateaux de l'Ouest (régions de Dschang et Bandjoun. (Bouopda, 2000 ; Tabué Youmbi, 2000 ; Momeny, 2000 ; Djomou Boupda, 2002 ; Wabo, 2002) sur socle et $\beta$ latéritisé et région des Bambouto (Biaya, 2002) sur $\beta$ et phonolitte relativement plus récents) dans le diagramme de Piper (1944), comparés aux faciès des eaux de la Ligne du Cameroun (Le Maréchal, 1974).

d'altération est dû à quelques facteurs physiques internes (température, $\mathrm{pH}$, degré d'aération) du milieu (eau-roche) et à la nature argileuse des minéraux secondaires qui facilitent les échanges ioniques avec l'eau. Les sources de la région des Bambouto (développées sur formations plus récentes et moins altérées) se rapprochent moins de la kaolinite (équilibre moins parfait) que les autres sources qui ont traversé des matériaux plus âgés et plus altérés.

Les équations chimiques suivantes montrent que certains éléments sont facilement mis en solution $\left(\mathrm{Na}^{+}, \mathrm{K}^{+}, \mathrm{Ca}^{2+}\right.$, $\left.\mathrm{Mg}^{2+}, \mathrm{H}_{4} \mathrm{SiO}_{4} \ldots\right)$ lors du contact eau - roche saine à travers les fissures.

$\begin{array}{ll}2 \mathrm{NaAlSi}_{3} \mathrm{O}_{8}+2 \mathrm{H}^{+}+9 \mathrm{H}_{2} \mathrm{O} \longrightarrow & \mathrm{Al}_{2} \mathrm{Si}_{2} \mathrm{O}_{5}(\mathrm{OH})_{4} \\ \text { Albite } & +4 \mathrm{H}_{4} \mathrm{SiO}_{4}+2 \mathrm{Na} \\ & \mathrm{Kaolinite}^{\longrightarrow} \\ 2 \mathrm{KAlSi}_{2} \mathrm{Si}_{2} \mathrm{O}_{5}(\mathrm{OH})_{4}+2 \mathrm{H}^{+}+9 \mathrm{H}_{2} \mathrm{O} \longrightarrow \\ \text { Orthose } & +4 \mathrm{H}_{4} \mathrm{SiO}_{4}+2 \mathrm{~K} \\ & \mathrm{Kaolinite}\end{array}$

$2 \mathrm{CaAl}_{2} \mathrm{Si}_{2} \mathrm{O}_{8}+\mathrm{H}_{2} \mathrm{O}+2 \mathrm{CO}_{2} \longrightarrow \mathrm{Al}_{2} \mathrm{Si}_{2} \mathrm{O}_{5}(\mathrm{OH})_{4}+$

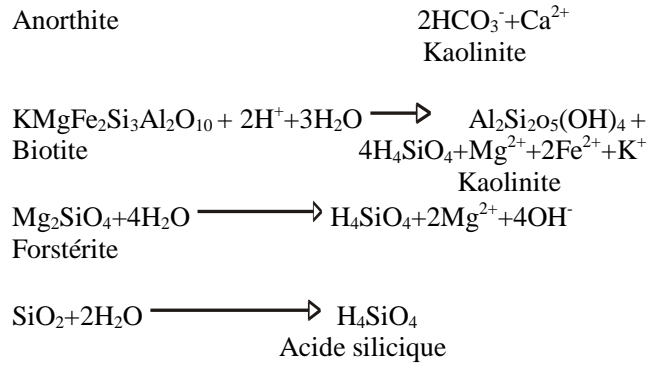

La minéralisation des eaux souterraines des Hauts Plateaux de l'Ouest bien que faible (moins de $587 \mathrm{mg} / \mathrm{l}$ ) semble liée aux faciès lithologiques; les valeurs les plus élevées de minéralisations se trouvant dans les basaltes et les plus faibles indifféremment dans les formations du socle que de la couverture volcanique. Cette différence dans la minéralisation peut s'expliquer par l'âge des formations, les plus âgées étant les plus lessivées. La minéralisation relativement plus élevée dans les forages (nappes profondes) peut certes s'expliquer par le séjour plus long 
des eaux, mais surtout par la quasi-inexistence de lessivage et d'adsorption des ions par les minéraux argileux, d'où leur mise en solution facile et leur forte concentration par rapport aux eaux des sources (nappes superficielles) (Nono et al., 2008).

Les ions présents dans les eaux souterraines des hauts plateaux de l'Ouest ont plusieurs origines. Les cations majeurs $\left(\mathrm{Na}^{+}\right.$, $\left.\mathrm{K}^{+}, \mathrm{Ca}^{2+}, \mathrm{Mg}^{2+}\right)$, la silice $\left(\mathrm{H}_{4} \mathrm{SiO}_{4}\right)$ et le fer proviennent de la roche saine (roche mère) selon les données géochimiques et les diagrammes d'équilibre. Le fer a des valeurs les plus élevées (3-4,70 mg/l) dans les orthogneiss et non dans les basaltes comme on se serait naturellement attendu. Les valeurs les plus élevées en silice (jusqu'à $112 \mathrm{mg} / \mathrm{l}$ ) se trouvent par contre plutôt dans les basaltes aphyriques et non dans les roches felsiques; cette concentration élevée en silice serait plutôt liée aux phénomènes d'hydrolyse dans les géodes (à cristallisation secondaire de quartz, calcite et zéolite) contenues dans les basaltes et non à leur chimie.

Les anions majeurs tels que le chlore proviendrait principalement des précipitations, l'ion $\mathrm{HCO}_{3}{ }^{-}$proviendrait du gaz carbonique $\left(\mathrm{CO}_{2}\right)$ des plantes et celui présent dans les profondeurs du sous-sol au contact de l'eau souterraine en circulation.
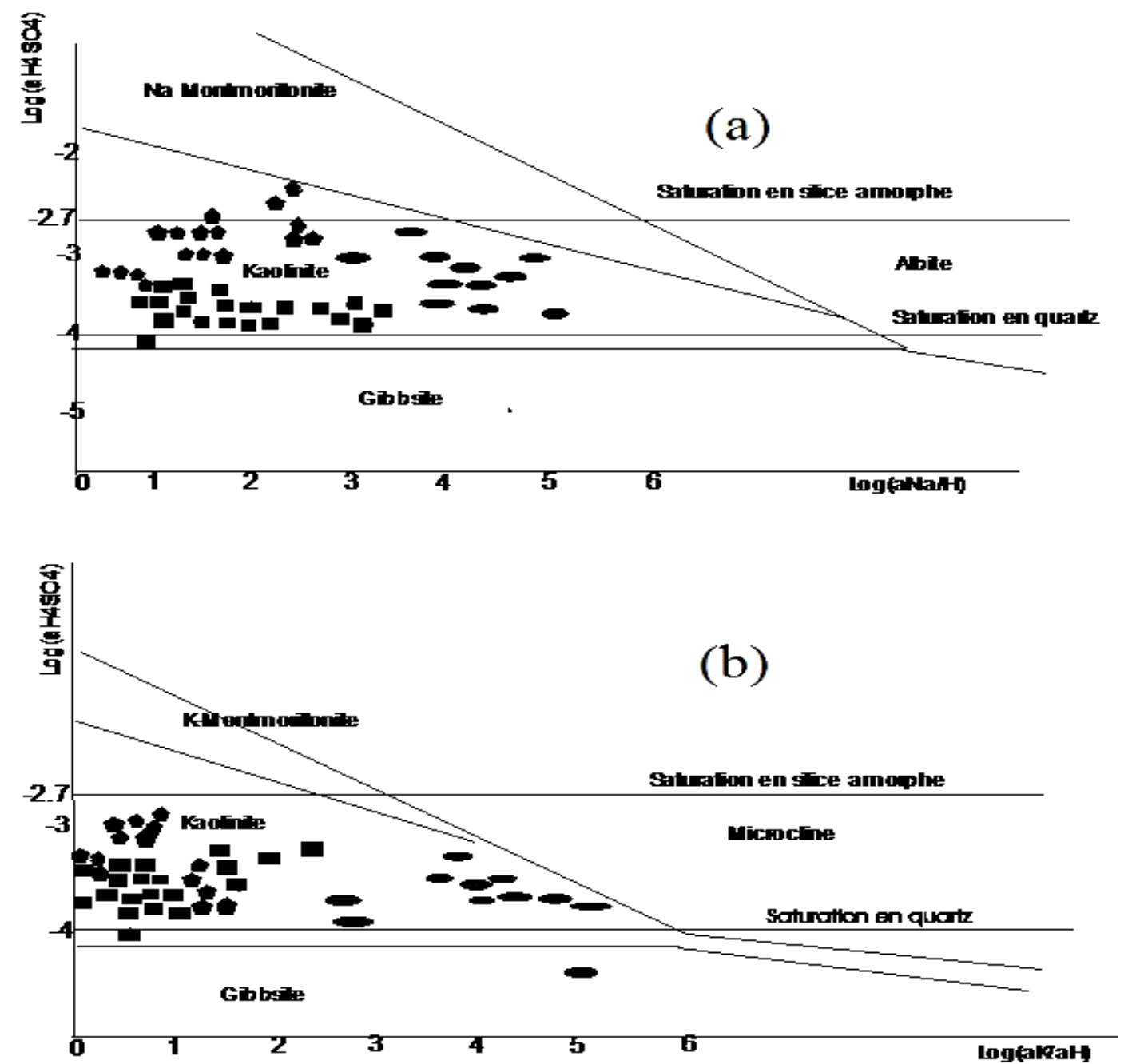

Figure 4: Position des eaux souterraines dans les diagrammes d'équilibre. a) Position des eaux souterraines dans le diagramme $\mathrm{Hcl}-\mathrm{H}_{2} \mathrm{O}-\mathrm{Al}_{2} \mathrm{O}_{3}-\mathrm{Na}_{2} \mathrm{O}-\mathrm{SiO}_{2}$. b) Position des eaux souterraines dans le diagramme $\mathrm{Hcl}-\mathrm{H}_{2} \mathrm{O}-$ $\mathrm{Al}_{2} \mathrm{O}_{3}-\mathrm{K}_{2} \mathrm{O}-\mathrm{SiO}_{2}$. 


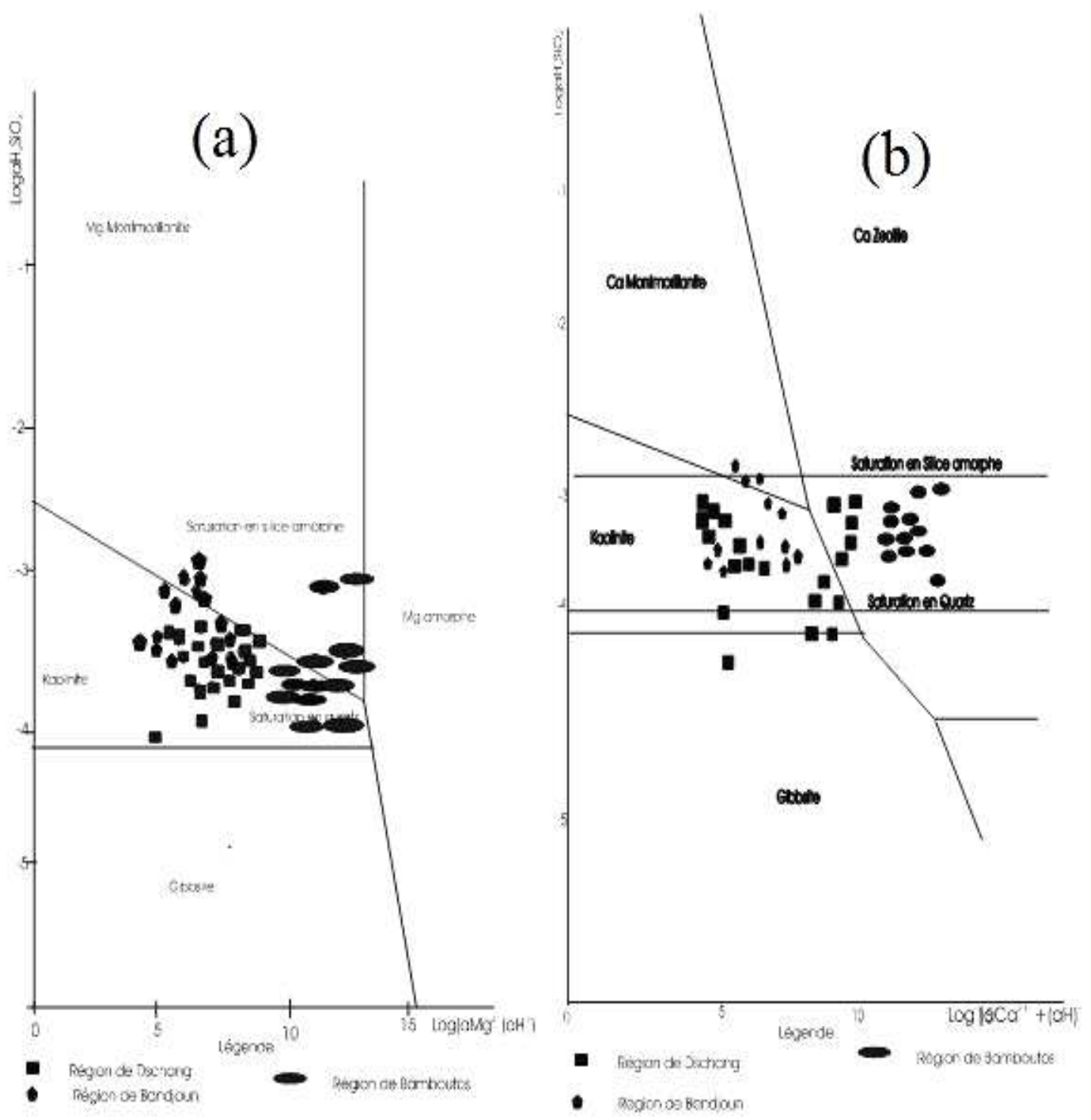

Figure 5: Position des eaux souterraines dans les diagrammes d'équilibre. a) Position des eaux souterraines dans le diagramme $\mathrm{Hcl}-\mathrm{H}_{2} \mathrm{O}-\mathrm{Al}_{2} \mathrm{O}_{3}-\mathrm{MgO}-\mathrm{SiO}_{2}$. b) Position des eaux souterraines dans le diagramme $\mathrm{Hcl}-\mathrm{H}_{2} \mathrm{O}-$ $\mathrm{Al}_{2} \mathrm{O}_{3}-\mathrm{CaO}-\mathrm{SiO}_{2}$

\section{Conclusion}

La quasi-totalité des eaux des sources étudiées sont chlorurées, calciques et magnésiennes, indépendamment de la nature et du degré d'altération de la roche-hôte. Les eaux des forages sont bicarbonatées calciques et magnésiennes. Les faciès chimiques des eaux ne semblent donc pas systématiquement liés aux faciès géologiques. La tendance bicarbonatée, sodique et potassique des sources thermominérales de la Ligne du Cameroun (Le Maréchal, 1974; Aka et al.,
2001) peut être attribuée à leur mode de mise en place où la présence des alcalins se justifie par leur grande solubilité (d'où leur extraction facile) et l'ion bicarbonate obtenu par transformation du $\mathrm{CO}_{2}$ magmatique (ou atmosphérique) au contact de l'eau souterraine.

Les minéralisations en général faibles sont plus ou moins liées à la nature lithologique du substrat. En effet les sources les plus minéralisées se trouvent dans les basaltes (soit 31\%), les 40 autres (soit 69\%) se 
trouvant dans le socle et le basalte, mais curieusement la source la moins minéralisée se trouve dans le basalte. De même, les forages les plus minéralisés (soit 50\%) se retrouvent dans les basaltes $(300 \mathrm{mg} / \mathrm{l}$ en moyenne), alors que les cinq autres qui ont été réalisés dans le socle sont les moins minéralisés.

Les eaux de forage présentent toutes des températures et des $\mathrm{pH}$ hors normes.

Tous les ions contenus dans les sources proviennent des roches-hôtes dans un processus de minéralisation en cours d'évolution comme le confirment le rapprochement sources-roches et l'éloignement de la courbe d'équilibre de Werner et Giggenbach (1988). Le fer bien connu pour son colmatage des canalisations et les dépôts boueux (favorisant le développement des bactéries), se trouve ici paradoxalement plus concentré dans les orthogneiss (jusqu'à 4,70 mg/l), les concentrations les plus faibles se trouvant indifféremment dans le socle et les basaltes.

L'âge des formations géologiques semble influencer les minéralisations des sources, car les sources des régions de Dschang et de Bandjoun sur socle et manteau volcanique très altérés ont le même degré d'équilibre avec la kaolinite, alors que les sources de la région des Bambouto sur matériau volcanique plus jeune et moins lessivé sont plus proches des minéraux primaires (microcline/albite) et plus riches en alcalino-terreux ( $\mathrm{Mg}$ et $\mathrm{Ca}$ amorphes).

L'incidence sur l'environnement et la santé humaine dans les hauts plateaux de l'OuestCameroun sera perceptible à cause des températures et des $\mathrm{pH}$ (dans les eaux de forages) et des teneurs de $\mathrm{Fe}, \mathrm{SiO}_{2}$ (dans les eaux des sources) non conformes aux normes de l'O.M.S. (Organisation Mondiale de la Santé), surtout dans les faciès basaltiques (naturellement riches en ferromagnésiens) où l'on note de nombreux géodes à cristallisation secondaire de silice fibreuse sous forme de calcédoine, de calcite et de zéolites, mais aussi dans certains orthogneiss.

\section{REFERENCES BIBLIOGRAPHIQUES}

Aka FT, Kusakabe M, Nagao K, Taniyleke G. 2001. Noble gaz isotipic composition and water/gaz chemistry of soda springs from the islands of Bioko, Sao Tomé and
Annobon, along the Cameroon volcanic Line, West Africa. Appl. Geochem., 16: 323-338.

Berthé D, Choukroune P, Jegouzo P.1979. Orthogneiss, mylonite and non coaxial deformation of granite: the example of South Armorican Shear Zone. Journal Struc. Geol., 1: 31-42.

Bessoles B, Trompette M. 1980. Géologie de l'Afrique : la chaîne panafricaine, «zone mobile d'Afrique Centrale (partie sud) et zone mobile soudanaise ». Mém. BRGM : Orléans, France.

Biaya SMO. 2002. Influences de la nature lithologique et des structures géologiques sur la qualité des eaux de sources : cas du village Balatchi et ses environs (Dans le Département des Bambouto) Mem. Maîtrise, Fac. Sc., Univ. Dschang, 88 p.

Bouopda JF. 2000. Influences de la nature lithologique et des structures géologiques sur la qualité des eaux de sources : cas de la région de Bandjoun. Mem. Maîtrise. Fac. Sc. Univ. Dschang, 87 p.

Derron MH. 1999. Interaction eau-roche de basse température: géochimie des métaux dans l'altération météorique des roches mafiques alpines. Thèse Doct., Fac. Sc., Univ. Lausanne, 128 p.

Deruelle B, Moreau C, Kambou R, Lissom J, Njonfang E, Ghogomu RT, Nono A. 1991. The Cameroon Line: a review. In Magmatism in Extentional Structural Settings. The Phanorozoïc African Plate, Kampuzu AB, Lubala RT (eds). Heidelberg Springer-Verlag; 237-274.

Djeuda THB. 1987. Géologie et hydrogéologie d'un secteur de la zone mobile d'Afrique Centrale: Région de poli, Nord Cameroun. Thèse. Doct., Univ. Grénoble I, 304 p. + annexes.

Djeuda THB, Tanawa E, Ngnigam E. 2001. L'eau au Cameroun: Approvisionnement en Eau Potable (Tome 1). Presses Universitaires: Yaoundé.

Djomou Bopda SL. 2002. Influence de la nature lithologique et des structures géologiques sur la qualité des eaux de sources: cas de la région de Dschang, et ses environs (Ouest-Cameroun). Mem. Maîtrise, Fac. Sc., Univ. Dschang, 75 p.

DNS, 1999. Direction Nationale de la Statistique 
Drever JI, Zobrist S. 1992. Chemical weathering of silicate rocks as a function of elevation in the southern Swiss Apls. In the Robert M. Garrels memorial issue. Helgeson HC (ed ); 56: 3209-3216.

Dubreuil P, Guiscarfe J, Nouvelot JF, Olivry JC. 1975. Le Bassin de la Rivière Sanaga. Monographies Hydrologiques ORSTOM, 3: Paris.

Dumort JC. 1968. Notice explicative sur la feuille Douala-Ouest avec carte géologique de reconnaissance au 1/500000. Dir. Min. Géol., Yaoundé, Cameroun.

Fosso J. 1999. Volcanologie, pétrographie et géochimie d'un strato-volcan des hauts plateaux de l'Ouest: le Mont Bangou. Thèse de Doct. $3^{\text {è }}$ cycle, Fac. Sc., Univ. Yaoundé I, 278 p.

Fosso J, Ménard JJ, Bardintzeff JM, Wandji P, Tchoua FM, Bellon H. 2005. Les laves du Mont Bangou : une première manifestation volvanique éocène, à affinité transitionnelle, de la Ligne du Cameroun. C. R. Geosciences, 337: 315325.

Furry V. 1997. Les eaux souterraines en Picardie. Mém. D.E.S.S. Environnement, Univ. Picardie Jules Verne, 30 p. + annexes.

Jackson TA. 1998. The biogeochemical and ecological significance of interactions between colloidal minerals and trace elements in environmental interactions of clays. Rae P (ed). Springer.

Kuepouo G. 2004. Geology, petrology and geochemistry of the Tertiary Bana volcano-plutonic complex, West Cameroon, Central Africa. PhD Thesis, Kobe University, Japan, $301 \mathrm{p}$.

Kuepouo G, Tchouankoue JP, Takashi N, Hiroaki S. 2006. Transitional tholeiitic basalts in the Tertiary Bana volcanoplutonic complex, Cameroon Line. Journ. Afric. Earth Sci., 45: 318-332.

Kwékam M. 1993. Le massif plutonique calco-alcalin pan-africain de Fomopéa (Ouest-Cameroun). Cadre structuralpétrologie-géochimie, interprétation géodynamique. Thèse Doct. $3^{\text {ème }}$ cycle, Univ. Yaoundé I, Cameroun, 283 p.

Kwékam M. 2005. Genèse et évolution des granitoïdes calco-alcalins au cours de la tectonique panafricaine: le cas des massifs syn- à tardi- tectoniques de l'Ouest-Cameroun (Régions de Dschang et de Kekem). Thèse de Doct. D'Etat, Université de Yaoundé I, 196 p.

Lasserre M. 1978. Mise au point sur les granitoîdes dits « ultimes » du Cameroun. Gisements, pétrographie et géochronologie. Bulletin du Bureau de Recherches Géologiques et Minières, 2(4): 143-159.

Le Maréchal A. 1974. Géologie et géochimie des sources thermoninérales du Cameroun. Thèse doct. D'Etat, Univ. Paris VI, ORSTOM PARIS, 165 p. + annexes.

Marzoli A, Renne PR., Picirillo ME, Castorina F, Giuliano B, Adolpho JM, Nyobe JB, Nni J. 1999. Slicic magmas from the continental Cameroon Volcanic Line (Oku, Bambouto and Ngaoundere) ${ }^{40} \mathrm{Ar}-{ }^{39} \mathrm{Ar}$ dates, petrology, $\mathrm{Sr}-\mathrm{Nd}-\mathrm{O}$ isotopes and their petrogenetic significance. Contib Minneral Petrol., 135: 133-150.

Marzoli A, Picirillo ME, Renne PR, Bellieni G, Iacumin M, Nyobe JB, Tongwa AT. 2000. The Cameroon Volcanic Line revisited: Petrogenesis of continental basaltic magmas from lithospheric and asthenospheric mantle sources. Journal of Petrology, 41(1): 87-109.

MINMEE/DRH, 1997. Note de service $\mathrm{N}^{\circ}$ 033/MINMEE/DRH/SDT/SES du 26 mars 1997 portant sur la situation du projet 400 forages dans sept provinces du Cameroun. Financement: Banque Islamique de Développement (BID), $1 \mathrm{p}$.

Momeny SR. 2000. Influence de la nature lithologique et des structures géologiques sur la qualité des eaux de sources : cas du secteur de Tchuelekwet-Mela-Tsinza dans les environs de Dschang (OuestCameroun). Mem. Maîtrise, Fac. Sc., Univ. Dschang, 66 p.

Moreau C, Regnoult JM, Déruelle B, Robineau B. 1987. A new tectonic model for the Cameroon Line, Central Africa. Tectonophysics, 139, 317-334.

Nahon D. 1976. Cuirasses ferrugineuses et encroûtement calcaires au Sénégal occidental et en Mauritanie. Système évolutifs: géochimie, structures, relais et coexistence. Sc. Géol. Mém. Strasbourg, 44. 
Nana JM. 1988. Le complexe volcanoplutonique de Bana (Ouest-Cameroun) : Géologie et pétrologie. Thèse de Doct. Univ. Paris Sud (Centre d'Orsay), 131 p.

Nguiessi Tchamkam C, Vialette Y. 1994. Données géochonologiques ( $\mathrm{Rb}-\mathrm{Sr}, \mathrm{Pb}$ $\mathrm{Pb}, \mathrm{U}-\mathrm{Pb}$ ) sur le complexe plutonique de Bandja (centre Ouest-cameroun). C.R. Acad. Sci., Paris, 315: 209-215.

Nguiessi Tchamkam C, Nzenti JP, Nsifa EN, Tempier P, Tchoua FM. 1997. Les granitoïdes calco-alcalins, syncisaillement de Bandja dans la chaîne panafricaine nord-équatoriale au Cameroun. C. R. Acad. Sc., 325: 95-101.

Njonfang E, Moreau C, Tchoua FM. 1998. La bande mylonitique Foumban-Bankim, Ouest-Cameroun. Une zone de cisaillement haute température. C.R. Acad. Sci., Paris, 327: 735-741.

Nni J, Nyobe JB. 1995. Géologie et pétrologie des laves pré-caldériques des Monts Bambouto: ligne du Cameroun. Geochimica Brasil, 9: 47-59.

Nono A, Djeuda THB, Njonfang E, Tella NFN, Tabué Youmbi J, Bouopda JF, 2001. Influence de la lithologie et des structures géologiques sur la qualité des eaux souterraines et de sources dans les hauts plateaux de l'Ouest-Cameroun. $12^{\text {th }}$ International Conference of the geological society of Africa .Geo environmental catastrophes in Africa. $J$. Geosc. Soc. Cameroon, 105-106.

Nono A, Nkouathio DG, Gountie DM, Njonfang E, Kagou DA, Tchoua FM. 2003. Zonal and vertical variations in welding rate and composition of ignimbrites of the Bambouto volcano (Cameroon Line, Central Afrca): volcanologocal importance. Geophy. Res. Absrt., 5: 14541.

Nono A, Njonfang E, Kagou DA, Nkouathio DG, Tchoua FM. 2004a. Pyroclastic deposits of the Bambouto volcano (Cameroon Line, Central Africa): evidence of an initial strombolian phase. Journ. Afr. Earth Sci., 39: 409-414.

Nono A, Wabo H, Biaya SMO. 2004b. Influences comparées de la lithologie et de la tectonique sur la dynamique et la chimie des eaux souterraines sur les hauts plateaux volcaniques de l'Ouest et sur le socle panafricain à l'Est du Cameroun. 20th Colloquium of African GeologyOrléans France, 02-07 Juin 2004, Abstracts volume, $318 \mathrm{p}$.

Nono A, Wabo H. 2004c. Influence de la lithologie et de la fracturation sur la dynamique et la chimie des eaux souterraines sur socle panafricain à l'Est du Cameroun: cas de la localité d'AbongMbang. 20th Colloquium of African Geology-Orléans France, 02-07 Juin 2004, Abst. volume, 317 p.

Nono A, Wabo H, Tabué Youmbi G, Biaya S, Ekodeck, GE. 2006. Influence of lithology and geologic structures on quality and dynamics of groundwater in Cameroon western highlands: a case study of Bandjoun village. Gulf of Guinea Geosciences Congress, November 8-10, Kribi-Cameroon, Abstracts volume, 60.

Nono A, Temgoua E, Likeng JDH, Djoukouo Tutchamo JP. 2008a. Influence de la nature lithologique et des structures géologiques sur la qualité des eaux souterraines sur le versant nord des Monts Bambouto (des hauts plateaux de l'OuestCameroun): cas du village Balépo et ses environs. A. G. R., Special Publication, 1 \& 2: 149-162.

Nono A, Likeng JDH. 2008b. Influences of lithology and geological structures on groundwater in volcanic areas of Cameroon: Cameroon Volcanic Line (CVL) and Adamawa plateau. International symposium on hydrogeology of volcanic rocks, 14-17 December 2008, Djibouti.

Nzenti JP, Bertrand SM, Macaudière J. 1994. La chaîne panafricaine au Cameroun: cherchons suture et modèle. $15^{\mathrm{e}}$ Réunion des Sciences de la Terre, Nancy, France, $99 \mathrm{p}$.

Nzenti JP, Tchoua FM. 1996. Les gneiss scapolatiques de le chaîne panafricaine nord équatoriale au Cameroun : témoins au Précambrien d'une sédimentation évaporitique en bordure nord du craton du Congo. C. R. Acad. Sc., 323: 289-294.

Nzolang C. 2005. Crustal evolution of the Precambrian basement in WestCameroon: inference from geochemistry, $\mathrm{Sr}-\mathrm{Nd}$ isotopes and experimental investigation of some granitoides and 
metamorphic rock. $\mathrm{PhD}$ Thesis, Niigata University, Japan, 207 p.

Peronne Y. 1969. Notice explicative de la feuille Wum-Banyo avec carte géologique de reconnaissance au 1/500000. Dir. Min. Géol., Yaoundé, Cameroun.

Piper AM. 1944. A graphic procedure in the geochemical interpretation of water analyses. Trans. Am. Geophys. Un. Papers, Hydrol. : 914-929.

Regnoult JM. 1986. Synthèse géologique du Cameroun. Ministère des Mines, de l'Eau et de l'Energie, Yaoundé, Cameroun.

Salbu B, Steinnes E. 1995. Trace Elements in Natural Water. C.R.C. Press: Inc.

Saviulo G, Secco L, Marzoli A, Piccirillo EM, Nyobé JB. 2000. Ca-rich pyroxene from basic to silisic volcanic rocks from the Cameroon Volcanic Line (West-Africa): crystal chemistry and petrological relationships. Miner. Prtrol., 79: 73-88.

Segalen H. 1967. Les sols et géomorphologie du Cameroun. Cah. ORSTOM, Série Pédol., 5(2): 137-187.

Tabué Youmbi JG. 2000. Influence de la nature lithologique et des structures géologiques sur la qualité des eaux de sources: cas du secteur Fokamezoung Doumbow (Sud-Est de la ville de Dschang, Ouest-Cameroun). Mem. Maîtrise, Fac. Sc., Univ. Dschang, 99 p.

Tagné Kamga G, Mercier E, Rossy M, Nsifa EN. 1999. Synkinematic emplacement of of the panafrican Ngondo igneous complex. (West-Cameron, central Africa). Journ. Afr. Earth Sci., 28: 675691.

Tagné Kamga G. 2003. Petrogenesis of the Neoproterozoic Ngondo plutonic complex (Cameron, West-central Africa): a case of late-collisional ferro-potassic magmatism. Journ. Afr. Earth Sci., 36: 149-171.

Talla V. 1995. Le massif granitique panafricain de Batié (Ouest-Cameroun): Pétrologie-Pétrostructurale-Géochimie. Thèse Doct. $3^{\text {ème }}$ cycle, Univ. Yaoundé I, Cameroun, $271 \mathrm{p}$.

Tchoua FM. 1968. Découverte d'ignimbrites dans la région de Dschang. Annales de la Faculté des Sciences Cameroun, 2: 77-94.
Tchoua FM. 1973. Sur l'existence d'une phase initiale ignimbritique dans le volcanisme des Monts Bambouto (Cameroun). C. R. Acad. Sc., 276: 28632866.

Tchouankoué JP. 1992. La syénite de Banganté: un complexe panfricain à caractères intermédiaires. PétrologieGéochimie. Thèse Doct. $3^{\text {ème }}$ cycle, Univ. Yaoundé I, Cameroun, 283 p.

Tella NFN. 1999. Contribution à l'étude hydrogéologique de la région des hauts plateaux de l'Ouest-Cameroun:cas de la région de Bandjoun. Thèse de M.Sc, F.A.S.A., Dschang, 110 p. + annexes.

Temgoua E, Djeuda THB, Tanawa E, Guenat C, Pfeifer HR. 2005. Groundwater fluctuations and footslope ferricrete soils in the humid tropical zone of southern Cameroon. Hydrol. Process, 19: 30973111.

Wabo H. 2002. Influences de la nature lithologique et des structures géologiques sur la qualité des eaux de sources : cas de la région de Bandjoun (Ouest-Cameroun). Mem. Maîtrise, Fac. Sc., Univ. Dschang, 78 p. + annexes.

Weeksteen G. 1957. Notice explicative sur la feuille Douala-Est avec carte géologique de reconnaissance au 1/500 000. Dir. Min. Géol., Yaoundé, Cameroun.

Werner D, Giggenbach F. 1988. Géothermal solute équilibra. Derivation of $\mathrm{Na}-\mathrm{K}-\mathrm{Mg}$ $\mathrm{Ca}$ geoindicator. Geochimica and Cosmochimica Acta., 52: 2749-2765.

Wouatong AL. 1998. Mineralogical study of weathering on the Bana complex, Western Part of Cameroon. Jour. Sci. Hiroshima, Ser. C., 11(1): 55-99.

Youmen D. 1994. Evolution volcanologique, pétrologique et temporelle de la caldéira des Mts Bambouto (Cameroun). Thèse Doc. Univ. Christiaan Albrecht. Kiel. Fac. Maths et Sci Nat, 274 p. +2 cartes.

Youmen D, Schmincke HU, Lissom J, Etame J. 2005. Données géochronologique: mise en évidence des différentes phases volcaniques au Miocène des Monts Bambouto (Ligne du Cameroun). Sci. Technol. Dev., 11(1): 49-57. 